\title{
Out-of-band and adjacent-channel interference reduction by analog nonlinear filters
}

\author{
Alexei V Nikitin ${ }^{1,2^{*}}$, Ruslan L Davidchack ${ }^{3}$ and Jeffrey E Smith ${ }^{4}$
}

\begin{abstract}
In a perfect world, we would have 'brick wall' filters, no-distortion amplifiers and mixers, and well-coordinated spectrum operations. The real world, however, is prone to various types of unintentional and intentional interference of technogenic (man-made) origin that can disrupt critical communication systems. In this paper, we introduce a methodology for mitigating technogenic interference in communication channels by analog nonlinear filters, with an emphasis on the mitigation of out-of-band and adjacent-channel interference.

Interference induced in a communications receiver by external transmitters can be viewed as wide-band non-Gaussian noise affecting a narrower-band signal of interest. This noise may contain a strong component within the receiver passband, which may dominate over the thermal noise. While the total wide-band interference seen by the receiver may or may not be impulsive, we demonstrate that the interfering component due to power emitted by the transmitter into the receiver channel is likely to appear impulsive under a wide range of conditions. We give an example of mechanisms of impulsive interference in digital communication systems resulting from the nonsmooth nature of any physically realizable modulation scheme for transmission of a digital (discontinuous) message.

We show that impulsive interference can be effectively mitigated by nonlinear differential limiters (NDLs). An NDL can be configured to behave linearly when the input signal does not contain outliers. When outliers are encountered, the nonlinear response of the NDL limits the magnitude of the respective outliers in the output signal. The signal quality is improved in excess of that achievable by the respective linear filter, increasing the capacity of a communications channel. The behavior of an NDL, and its degree of nonlinearity, is controlled by a single parameter in a manner that enables significantly better overall suppression of the noise-containing impulsive components compared to the respective linear filter. Adaptive configurations of NDLs are similarly controlled by a single parameter and are suitable for improving quality of nonstationary signals under time-varying noise conditions. NDLs are designed to be fully compatible with existing linear devices and systems and to be used as an enhancement, or as a low-cost alternative, to the state-of-art interference mitigation methods.
\end{abstract}

Keywords: Adjacent-channel interference; Impulsive noise; Interchannel interference; Spectral density; Nonlinear differential limiters; Nonlinear filters; MANET; Out-of-band interference; Technogenic noise

\section{Introduction}

In a utopian world, our communication technology would have 'brick wall' filters, no-distortion amplifiers and mixers, and well-coordinated spectrum operations. In the real world, wireless communications are prone to various types of natural and technogenic (manmade) interference. Over the years, engineers developed

\footnotetext{
*Correspondence: avn@avatekh.com

${ }_{1}^{1}$ Avatekh Inc., 901 Kentucky Street, Suite 303, Lawrence, KS 66044, USA

2 Dept. of Electrical and Computer Engineering, Kansas State University,

Manhattan, KS 66506, USA

Full list of author information is available at the end of the article
}

effective filters and approaches to dealing with natural interference, but the need to transmit more and more data leads to ever-increasing levels of technogenic interference as we saturate the information-carrying capacity of the electromagnetic spectrum. This brings the understanding of the types of technogenic interference and development of effective ways of its mitigation to the forefront of challenges facing modern communication technology.

Technogenic noise comes in a great variety of forms, but it will typically have a temporal and/or amplitude structure which distinguishes it form the natural (e.g., thermal) noise. It will typically also have non-Gaussian amplitude

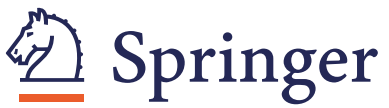


distribution. These features of technogenic noise provide an opportunity for its mitigation by nonlinear filters, especially for the in-band noise, where linear filters that are typically deployed in the communication receiver (see top part of Figure 1) have very little or no effect. Indeed, at any given frequency, a linear filter affects both the noise and the signal of interest proportionally. When a linear filter is used to suppress the interference outside of the passband of interest, the resulting signal quality is affected by the total power and spectral composition, but not by the type of the amplitude distribution of the interfering signal. On the other hand, the spectral density of a non-Gaussian interference in the signal passband can be reduced, without significantly affecting the signal of interest, by introducing an appropriately chosen feedbackbased nonlinearity into the response of the linear filter.

In particular, impulsive interference that is characterized by a frequent occurrence of outliers (i.e., relatively high-amplitude, short-duration events) can be effectively mitigated by the nonlinear differential limiters (NDLs) described in [1-4] and in Section 3 of this paper. An NDL can be configured to behave linearly when the input signal does not contain outliers, but when the outliers are encountered, the nonlinear response of the NDL limits the magnitude of the respective outliers in the output signal. As a result, the improvement in signal quality achieved by the NDL exceeds that achievable by the respective linear filter, increasing the capacity of a communications channel. Even if the interference appears nonimpulsive, the non-Gaussian nature of its amplitude distribution enables simple analog pre-processing which can increase its peakedness and thus increases the effectiveness of the NDL mitigation.

Another important consideration is the dynamic nonstationary nature of technogenic noise. When the frequency bands, modulation/communication protocol schemes, power levels, and other parameters of the transmitter and the receiver are stationary and well defined, the interference scenarios may be analyzed in great detail. Then, the system may be carefully engineered (albeit at a cost) to minimize the interference ${ }^{a}$. It is far more challenging to quantify and address the multitude of complicated interference scenarios in nonstationary communication systems such as, for example, software-defined

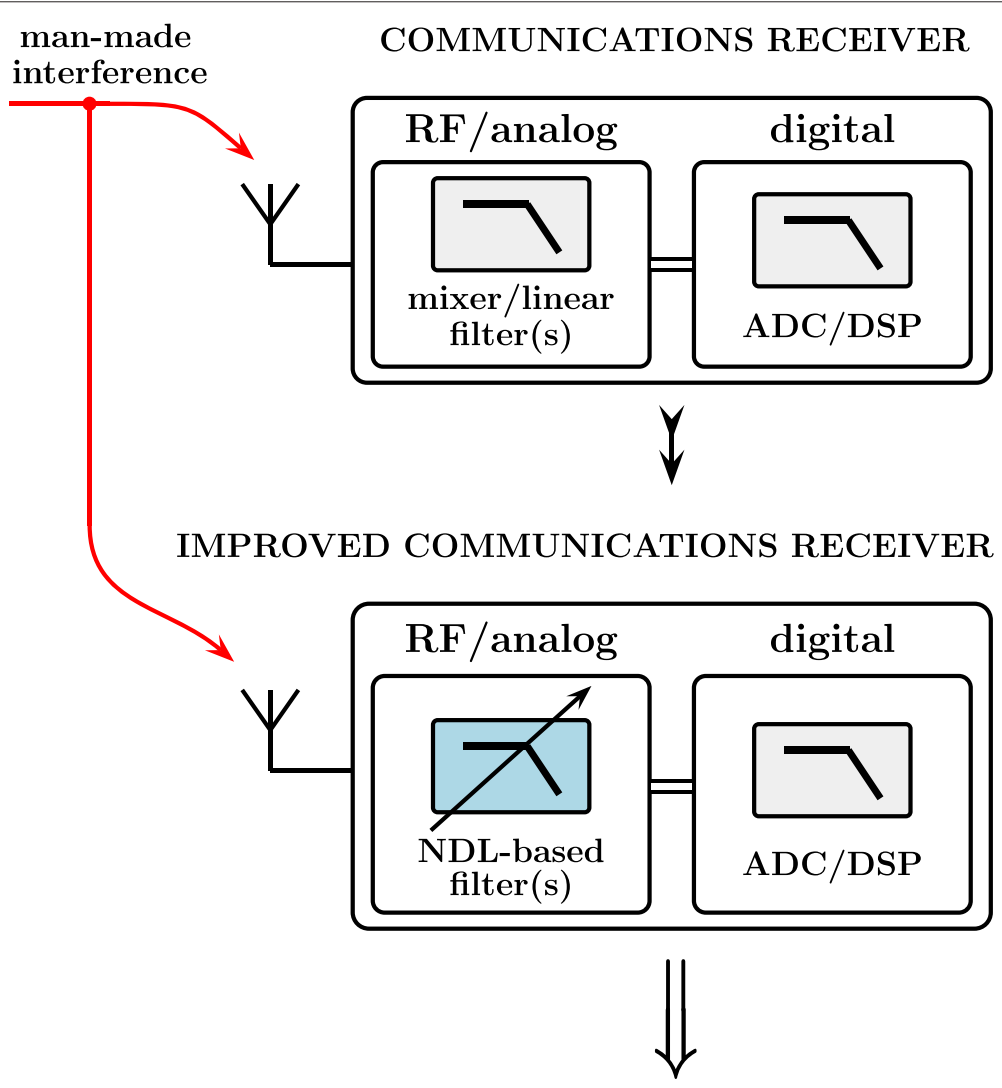

INCREASED CHANNEL CAPACITY

in the presence of man-made interference

Figure 1 Simplified diagram of improving receiver performance in the presence of technogenic (man-made) interference. 
radio (SDR)-based and cognitive ad hoc networks comprising mobile transmitters and receivers, each acting as a local router communicating with a mobile ad hoc network (MANET) access point [5]. In this scenario, the transmitter positions, powers, and/or spectrum allocations may vary dynamically. In multiple access schemes, the interference is affected by the varying distribution and arrangement of transmitting nodes [6]. In addition, with MANETs, the fading distribution also varies dynamically, and the path loss distribution is unbounded [7]. With spectrum-aware MANETs, frequency allocations could also depend on various criteria, e.g., whitespace and the customer quality of service goals [8]. This is a very challenging situation which requires interference mitigation tools to adapt to the dynamically changing interference. Following the dynamic nature of the ad hoc networks, where the networks themselves are scalable and adaptive, and include spectrum sensing and dynamic re-configuration of the network parameters, interference mitigation tools are needed to be scalable and adaptive to the dynamically changing interference. Adaptive NDLs (ANDLs) [2,3] have been developed to address this challenge.

Based on the above considerations, we propose a modification of a communications receiver system as illustrated in the bottom part of Figure 1, where an NDL or ANDL filter is used as a replacement, or in conjunction with the existing mixer/linear filters.

To give more specificity to our presentation, let us consider a single transmitter-receiver system. Figure 2 provides a simplified qualitative illustration of different contributions into the interference which a receiver (RX) experiences from a transmitter (TX). Since realtime 'brick-wall' filters are not physically realizable as they have infinite latency (i.e., their compact support in the frequency domain forces their time responses not to have compact support, meaning that they are everlasting) and infinite order (i.e., their responses cannot be expressed as a linear differential equation with a finite sum), TX emissions would 'leak' outside of the nominal (allocated) passband of a TX channel $\left[f_{1}, f_{2}\right]$ as out-ofband (OOB) emissions. Likewise, an RX filter would have nonzero response outside of its nominal (allocated) passband $\left[f_{3}, f_{4}\right]$. As a result, there is nonzero interference from the TX into the RX.

The total power of this interference may be broken into three parts. Part I is the power of the TX signal in its nominal band $\left[f_{1}, f_{2}\right]$, weighted by the response of the RX filter in this band. Part II is the TX OOB emissions in the RX nominal band $\left[f_{3}, f_{4}\right]$, weighted by the response of the RX filter in this band. The rest of the interference power comes from the TX emissions outside of the nominal bands of both channels and can be normally ignored in practice since in those frequency regions both the emitted TX power and the RX filter response would be relatively small.

While part I of the interference contributes into the total power in the RX channel and may cause RX overload, it does not normally degrade the quality of the communications in the RX since the frequency content of this part of the interference lies outside of the RX channel. Part II, however, in addition to contributing to overload, also causes degradation in the RX communication signal as it raises the noise floor in the RX channel.

Theoretical $[9,10]$ as well as the experimental [11] data suggests that the TX OOB interference in the RX channel (part II of the interference in Figure 2) can appear impulsive under a wide range of conditions, as will be additionally illustrated in Section 2. While this interference cannot be reduced by the subsequent linear filtering in the RX channel, it may be effectively mitigated by the NDLs introduced in Section 3.

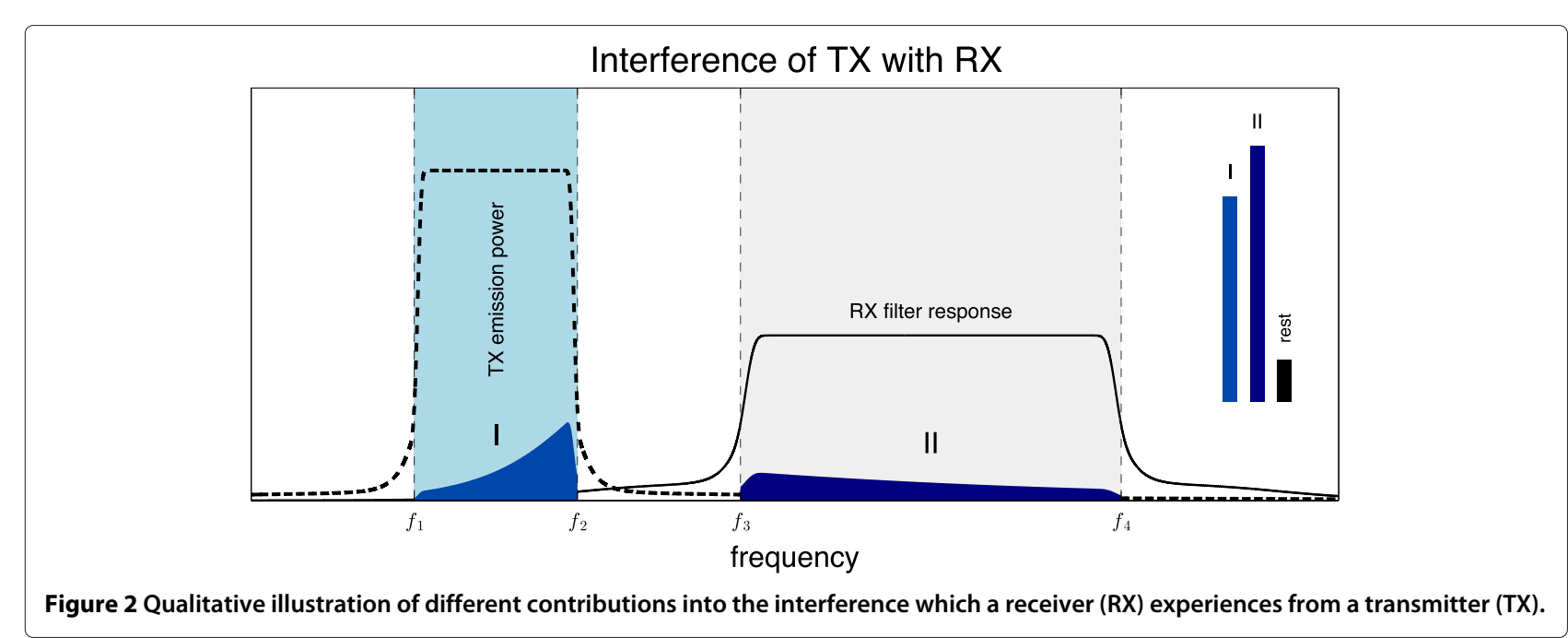


In Section 4, we show that an NDL deployed in the RX channel can reduce the spectral density of impulsive interference in the signal passband without significantly affecting the signal of interest, thus improving the baseband signal-to-noise ratio and increasing the channel capacity. We also show that, when part I of the interference in Figure 2 dominates over part II and the total interference observed in the receiver does not appear impulsive, one can deploy a bandstop linear filter in the signal chain of the receiver preceding the NDL to suppress part I of the interference without affecting the baseband signal of interest. By suppressing part I of the interference and thus increasing the peakedness of the remaining interference affecting the baseband signal, this additional filter can greatly improve the effectiveness of interference mitigation by the subsequently deployed NDL.
In Section 5, we provide some concluding remarks and comment on the possibility of digital implementations and deployment of the NDLs.

\section{Impulsive nature of interchannel interference}

As shown in more detail in $[9,10]$, with additional experimental evidence presented in [11], the signal components induced in a receiver by out-of-band communication transmitters can appear impulsive under a wide range of conditions. For example, in the transmitter-receiver pair schematically shown at the top of Figure 3, for a sufficiently large absolute value of the difference between the transmit and receive frequencies $\Delta f=f_{\mathrm{RX}}-f_{\mathrm{TX}}$, the instantaneous power $I^{2}(t, \Delta f)+Q^{2}(t, \Delta f)$ of the in-phase and quadrature components of the receiver signal may appear as a train of pulses consisting of a linear combination of pulses originating at discrete times and shaped
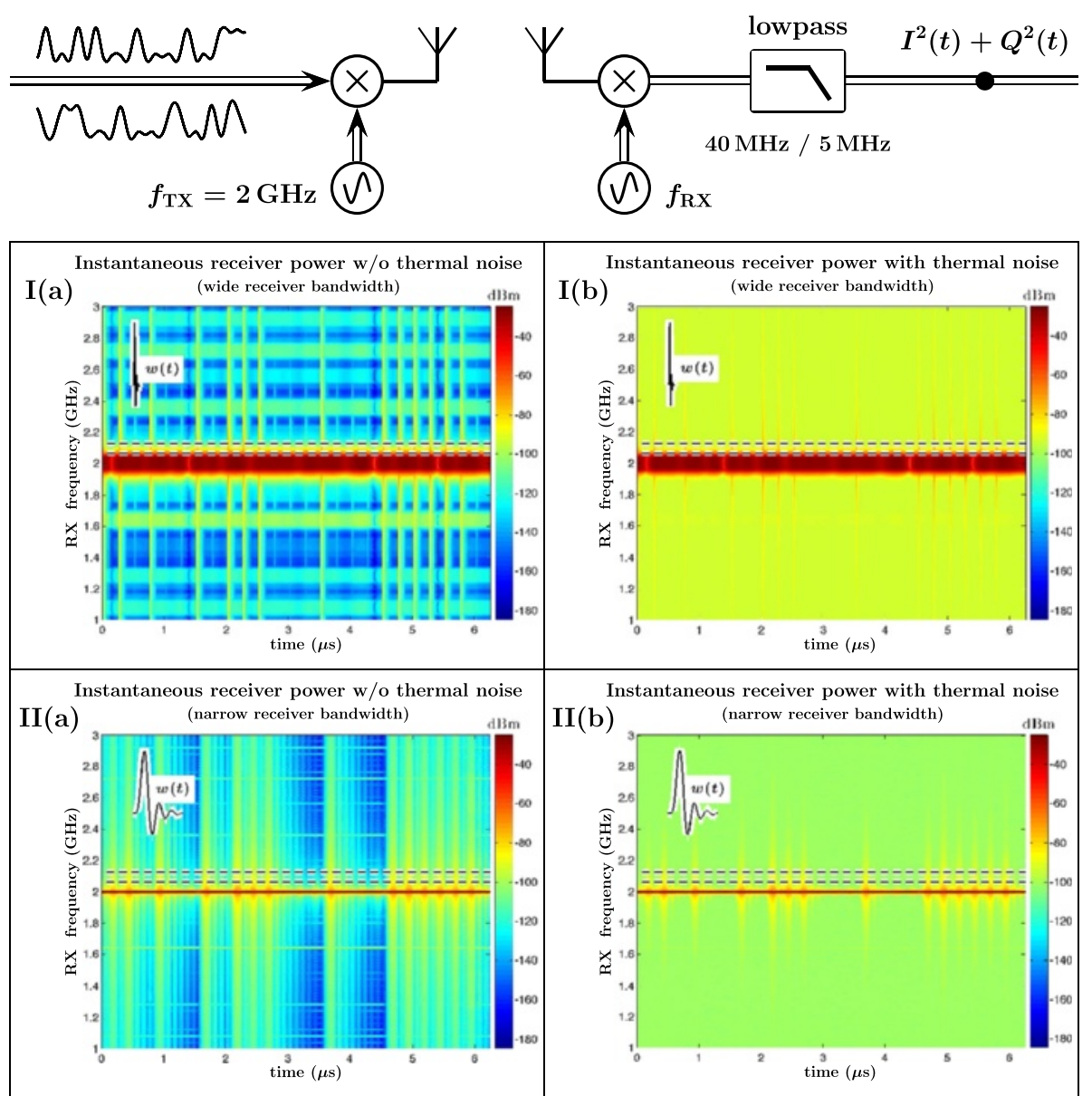

Figure 3 Instantaneous power response of quadrature receiver tuned to the RX frequency $f_{\mathrm{RX}}$ (in the 1- to 3 -GHz range). The transmitted signal is in a $5-\mathrm{MHz}$ band around $2 \mathrm{GHz}$, the transmit power is $125 \mathrm{~mW}(21 \mathrm{dBm})$, and the path/coupling loss is $50 \mathrm{~dB}$. Panels $\mathbf{I}(\mathbf{a})$ and $\mathbf{I}(\mathbf{b})$ : wide bandwidth of the lowpass filter (40-MHz eighth-order Butterworth filter) without (panel I(a)) and with (panel I(b)) thermal noise. Panels II(a) and $\mathbf{I I}(\mathbf{b})$ : narrow bandwidth of the lowpass filter (5-MHz eighth-order Butterworth filter) without (panel II(a)) and with (panel II(b)) thermal noise. The impulse response (time window) $w(t)$ of the lowpass filter is shown in the upper left corners of the respective panels. 
as the squared (i.e., raised to the power of 2) impulse response of the receiver lowpass filter.

For a single transmitter, the typical intervals between those discrete times are multiples of the symbol duration of the transmitted signal (or other discrete time intervals used in the designed modulation scheme, for example, chip and guard intervals). The nonidealities in hardware implementation of designed modulation schemes, such as the nonsmooth behavior of the modulator around zero, and/or nonlinearities in the power amplifier, can also lead to the appearance of additional discrete origins for the pulses and exacerbate the $\mathrm{OOB}$ emissions. If the typical value of those discrete time intervals is large in comparison with the inverse bandwidth of the lowpass filter in the receiver, this pulse train may be highly impulsive.

A key mathematical argument leading to this conclusion can be briefly recited as follows $[9,10]$. The total emission from various digital transmitters can be written as a linear combination of the terms of the following form:

$$
x(t)=A_{T}(\bar{t}) \mathrm{e}^{\mathrm{i} \omega_{\mathrm{c}} t},
$$

where $\omega_{\mathrm{c}}$ is the frequency of a carrier, $\bar{t}=\frac{2 \pi}{T} t$ is the nondimensionalized time, and $A_{T}(\bar{t})$ is the desired (or designed) complex-valued modulating signal representing a data signal with symbol duration (unit interval) $T$. Let us assume that, for some integer $n$, all derivatives of order smaller than $n-1$ of the modulating signal $A_{T}(\bar{t})$ are finite, but the derivative of order $n-1$ of $A_{T}(\bar{t})$ has a countable number of step discontinuities ${ }^{\mathrm{b}}$ at $\left\{\bar{t}_{i}\right\}$. Let us now assume that the impulse response of the lowpass filters in both channels of a quadrature receiver is $w(t)=\frac{2 \pi}{T} h(\bar{t})$, and that the order of the filter is larger than $n$, so that all derivatives of $w(t)$ of order smaller or equal to $n-1$ are continuous. ${ }^{\mathrm{c}}$ Then, if $\Delta \omega=2 \pi \Delta f$ is the difference between the receiver and the carrier frequencies, and the bandwidth of the lowpass filter $w(t)$ in the receiver is much smaller than $|\Delta f|$, the instantaneous power in the quadrature receiver due to $x(t)$ can be expressed as ${ }^{\mathrm{d}}$ :

$$
\begin{aligned}
P_{x}(t, \Delta f)= & \frac{1}{(T \Delta f)^{2 n}} \sum_{i} \alpha_{i} h\left(\bar{t}-\bar{t}_{i}\right) \sum_{j} \alpha_{j}^{*} h\left(\bar{t}-\bar{t}_{j}\right) \\
& \text { for } \quad T \Delta f \gg 1,
\end{aligned}
$$

where $\alpha_{i}$ is the value of the $i$ th discontinuity of the order $n-1$ derivative of $A_{T}(\bar{t})$ :

$$
\alpha_{i}=\lim _{\varepsilon \rightarrow 0}\left[A_{T}^{(n-1)}\left(\bar{t}_{i}+\varepsilon\right)-A_{T}^{(n-1)}\left(\bar{t}_{i}-\varepsilon\right)\right] \neq 0 .
$$

The detailed derivation of Equation 2 can be found in $[9,10]$.
When viewed as a function of both time and frequency, the interpretation of Equation 2 for the instantaneous power in a quadrature receiver is a spectrogram ([12], for example) in the time window $w(t)$ of the term $x(t)$ of the transmitted signal. Figure 3 provides an illustrative example of such spectrograms for the $I^{2}(t, \Delta f)+Q^{2}(t, \Delta f)$ receiver signal in the transmitter-receiver pair schematically shown at the top of the figure.

The spectrograms displayed in the panels of the figure show the instantaneous power response of a quadrature receiver tuned to the RX frequency $f_{\mathrm{RX}}$, where $f_{\mathrm{RX}}$ is in the 1 - to $3-\mathrm{GHz}$ range. The transmitted signal is in a $5-\mathrm{MHz}$ band around $2 \mathrm{GHz}$, the transmit power is $125 \mathrm{~mW}(21 \mathrm{dBm})$, and the path/coupling loss is $50 \mathrm{~dB}$. A more detailed description of the simulation parameters used in Figure 3 and the subsequent examples can be found in Appendix A.

In panels $I(a)$ and $I(b)$ of Figure 3, the bandwidth of the lowpass filter (eighth-order Butterworth) is $40 \mathrm{MHz}$ (wide), while in panels $\mathrm{II}(\mathrm{a})$ and $\mathrm{II}(\mathrm{b})$, it is $5 \mathrm{MHz}$ (narrow). Panels I(a) and II(a) show the receiver power due to the transmitter signal only (without thermal noise), while panels I(b) and II(b) show the receiver power with additive white Gaussian noise (AWGN) taken as the thermal noise multiplied by the noise figure of the receiver (assumed $5 \mathrm{~dB}$ ). The shape of the impulse response (time window) $w(t)=\frac{2 \pi}{T} h(\bar{t})$ of the lowpass filters is shown in the upper left corners of the respective panels. The dashed horizontal lines in the panels indicate the specific receiver offset frequencies $\Delta f=65 \mathrm{MHz}$ and $\Delta f=125 \mathrm{MHz}$ used in the subsequent examples. To make the OOB interference induced by the transmitter less idealized, moderate intermodulation (resulting from 'clipping' of the carrier signal at high amplitudes) was added to the simulation. This results in the intermodulation distortion (IMD) that appears as horizontal bands at frequencies different from the carrier frequency in panels I(a) and II(a).

The upper panels of Figure 4 show the instantaneous receiver power averaged over time, for both wide (blue lines) and narrow (red lines) bandwidths of the lowpass filter in the receiver. These would be akin to the power spectra obtained by a spectrum analyzer with the resolution bandwidth (RBW) filters of $5 \mathrm{MHz}$ (red line) and $40 \mathrm{MHz}$ (blue line), without (left panels) and with (right panels) thermal noise taken into account.

Obviously, the average receiver power as a function of the RX frequency does not provide information on the peakedness of the receiver signal. The lower panels of Figure 4 quantify such peakedness of the receiver signal $z(t)=I(t)+\mathrm{iQ}(t)$ in terms of the measure $K_{\mathrm{dBG}}$ found in $[2,3]$ :

$$
K_{\mathrm{dBG}}(z)=10 \lg \left(\frac{\left\langle|z|^{4}\right\rangle-|\langle z z\rangle|^{2}}{2\left\langle|z|^{2}\right\rangle^{2}}\right),
$$




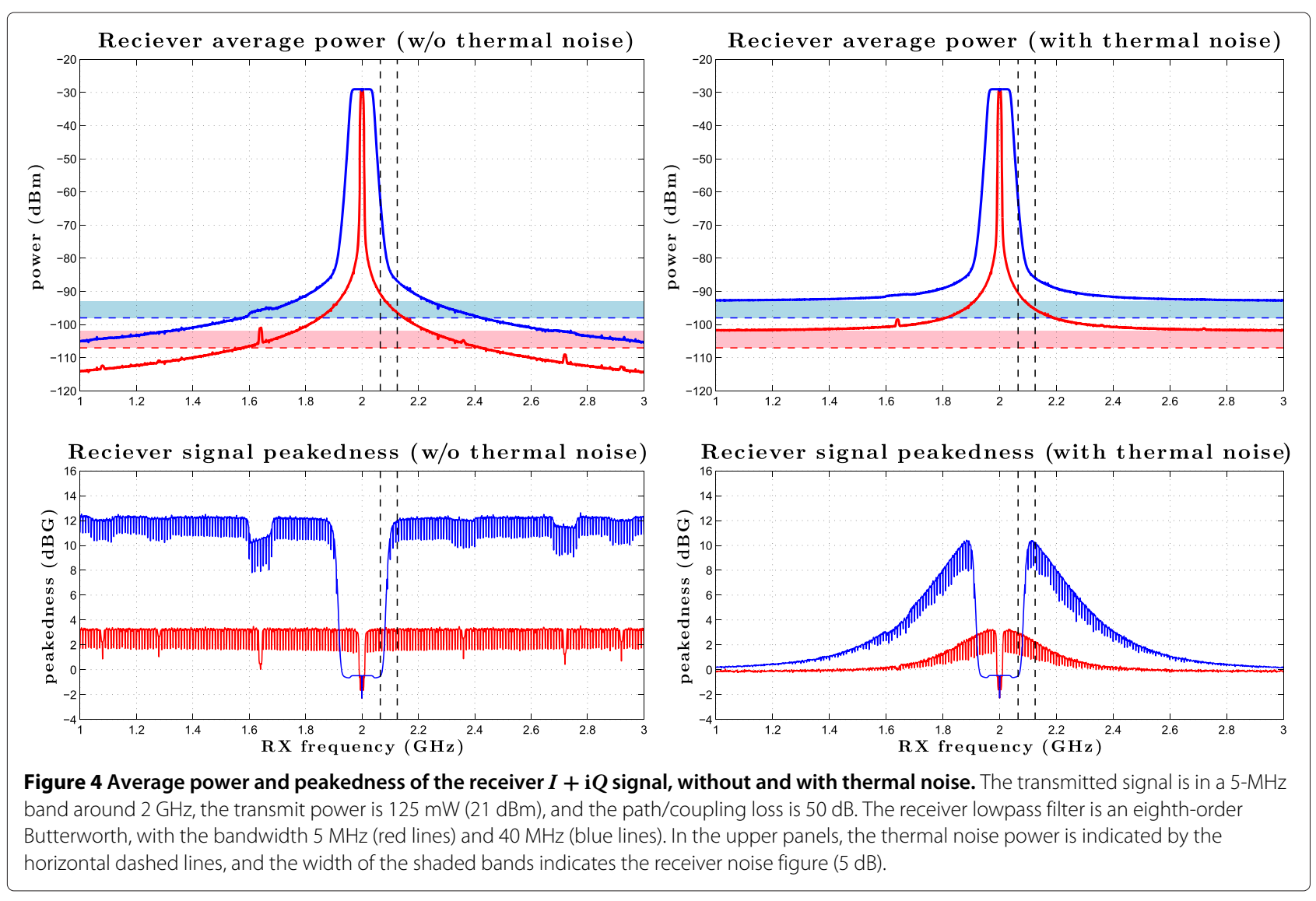

where the angular brackets denote time averaging. This measure of peakedness is based on an extension of the classical definition of kurtosis ([13], for example) to complex variables ([14], for example). According to this definition, the peakedness is measured in units of 'decibels relative to Gaussian' (dBG) (i.e., in relation to the kurtosis of the Gaussian (aka normal) distribution). Gaussian distribution has zero dBG peakedness, while sub-Gaussian and super-Gaussian distributions have negative and positive $\mathrm{dBG}$ peakedness, respectively.

As can be seen in the lower left panel of Figure 4, the peakedness of the receiver $I+\mathrm{i} Q$ signal at large values of $|\Delta f|$ is much higher for the wide-bandwidth receiver (blue line) than for the narrow-band receiver (red line). As follows from the linearity property of kurtosis, adding a Gaussian (zero dBG) signal to a super-Gaussian (positive $\mathrm{dBG}$ ) signal would lower the peakedness of the mixture. This can be seen in the lower right panel of Figure 4, where the peakedness remains high while the power of the $\mathrm{OOB}$ interference dominates over the thermal noise, asymptotically approaching zero as the $\mathrm{OOB}$ interference decays at large values of $|\Delta f|$.

Figure 5 provides time (upper panel) and frequency (lower panel) domain quantification of the receiver $I+\mathrm{i} Q$ signal without thermal noise for $\Delta f=125 \mathrm{MHz}$ and wide (blue lines) and narrow (red lines) bandwidths of the lowpass filter.

As discussed in Section 2.1, for a receiver lowpass filter of a given type and order, the amplitude ('height') of the interference pulses would be proportional to the bandwidth of the filter. This can be seen in the upper panel of Figure 5, where the peak amplitude of the pulses shown by the blue lines $(40-\mathrm{MHz}$ filter) is eight times the peak amplitude of the pulses shown by the red lines $(5-\mathrm{MHz}$ filter).

Since the duration of the pulses is inversely proportional to the lowpass filter bandwidth, the time average of the squared amplitudes of the pulses would be proportional to the bandwidth, while the average of the amplitudes raised to the fourth power would be proportional to the bandwidth raised to the third power. As a result, the measure of peakedness given by Equation 4 would be approximately proportional to a logarithm of the bandwidth. Thus, the increase in the bandwidth of the receiver lowpass filter from 5 to $40 \mathrm{MHz}$ (by $9 \mathrm{~dB}$ ) would result in a $9-\mathrm{dB}$ increase of peakedness. This is confirmed by the measured values of peakedness indicated in the lower panel of Figure 5 for the $5-\mathrm{MHz}$ bandwidth filter (3.2 dBG, red text) and the $40-\mathrm{MHz}$ bandwidth filter (12 dBG, blue text). 

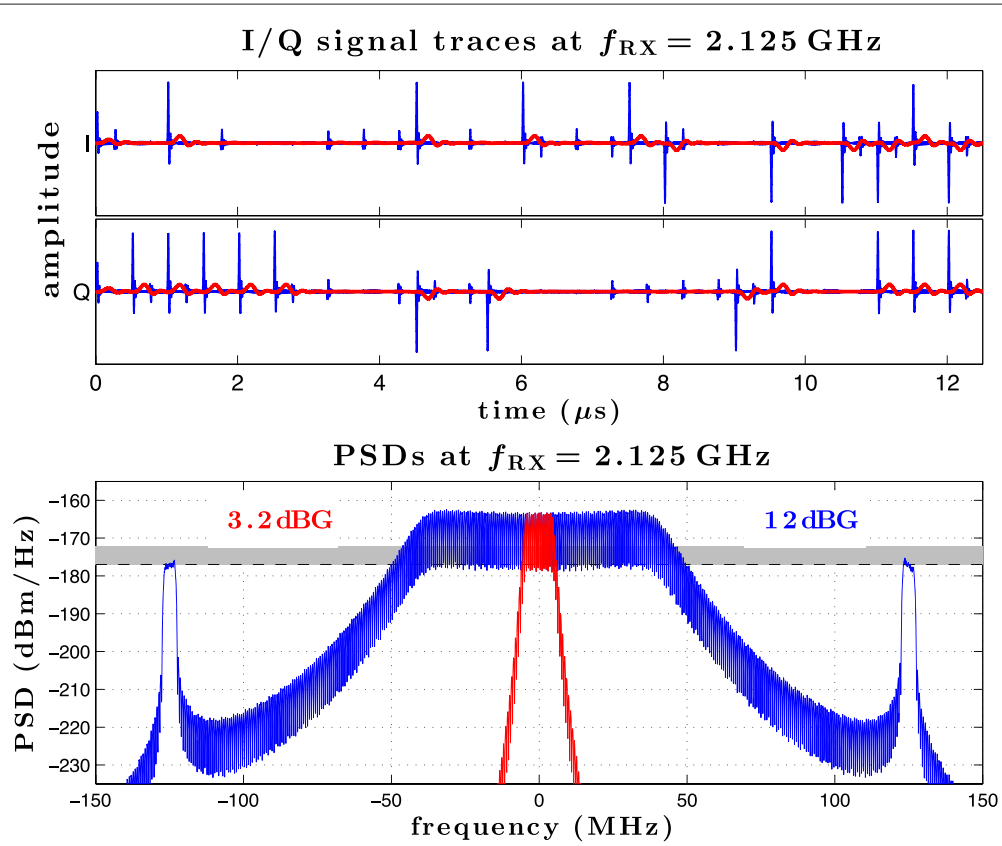

Figure 5 Time and frequency domain quantification of receiver signal without thermal noise at wide and narrow bandwidths ( $\boldsymbol{\Delta} \boldsymbol{f}=\mathbf{1 2 5} \mathbf{~ M H z}$ ). Upper panel: in-phase/quadrature (I/Q) signal traces for $f_{\mathrm{RX}}=2.125 \mathrm{GHz}$ and the receiver lowpass filters $5 \mathrm{MHz}$ (red lines) and $40 \mathrm{MHz}$ (blue lines). Lower panel: power spectral densities and peakedness of the receiver signal for the receiver lowpass filters $5 \mathrm{MHz}$ (red) and $40 \mathrm{MHz}$ (blue). The thermal noise density is indicated by the horizontal dashed line. The width of the shaded band indicates the receiver noise figure $(5 \mathrm{~dB})$.

Not surprisingly, as can be seen in the lower panel of Figure 5, the power spectral density (PSD) of the interference around $\Delta f=0$ (in baseband) is identical for both wide- and narrow-bandwidth receiver filters, and if subsequent linear processing is used (e.g., the signal is digitized and filtered with a matching digital filter), the resulting signal quality is independent of the bandwidth of the lowpass filter in the receiver. However, while the increase in the bandwidth of the receiver lowpass filter does not affect the baseband PSD of either the interference or the thermal noise, widening this bandwidth increases the peakedness of the interference, enabling its more effective mitigation by the NDLs introduced in Section 3.

Figure 6 provides time (upper panel) and frequency (lower panel) domain quantification of the receiver $I+\mathrm{i} Q$ signal without thermal noise for $\Delta f=65 \mathrm{MHz}$, for a 40$\mathrm{MHz}$ lowpass filter (green lines) and for a 40-MHz lowpass filter cascaded with a $65-\mathrm{MHz}$ notch filter (black lines).

The response of the receiver $40-\mathrm{MHz}$ lowpass filter at $65 \mathrm{MHz}$ is relatively large, and as can be seen in both panels of Figure 6 (green lines and text), the contribution of the TX signal in its nominal band (part I of the interference in Figure 2) into the total interference becomes significant, reducing the peakedness of the total interference and making it sub-Gaussian ( $-0.5 \mathrm{dBG}$ peakedness). However, since the sub-Gaussian part of the interference lies outside of the baseband, cascading a $65-\mathrm{MHz}$ notch filter with the lowpass filter would reduce this part of the interference without affecting either the signal of interest or the PSD of the impulsive interference around the baseband. Then, as shown by the black lines and text in Figure 6, the interference becomes super-Gaussian (10.8-dBG peakedness), enabling, as illustrated further in Section 4, its effective mitigation by the NDLs.

\subsection{Effects of symbol rates and pulse shaping on the interference power}

When the origins of the OOB interference lie in the finite duration of the finite impulse response (FIR) filters used for pulse shaping, an average value of $t_{i+1}-t_{i}$ in Equation 2 is of the same order of magnitude as the symbol duration (unit interval) $T$ (in the range from $T / 2$ to $T$, and equal to $T$ if the group delay is a multiple of $T$ ). If the reciprocal of this value (the symbol rate) is small in comparison with the bandwidth of the receiver, the contribution of the terms $\alpha_{i} \alpha_{j}^{*} h\left(\bar{t}-\bar{t}_{i}\right) h\left(\bar{t}-\bar{t}_{j}\right)$ for $i \neq j$ is negligible, and Equation 2 describes an impulsive pulse train consisting of a linear combination of pulses shaped as $w^{2}(t)$ and originating at $\left\{t_{i}\right\}$, namely:

$$
P_{x}(t, \Delta f)=\frac{1}{(T \Delta f)^{2 n}} \sum_{i}\left|\alpha_{i}\right|^{2} h^{2}\left(\bar{t}-\bar{t}_{i}\right)
$$



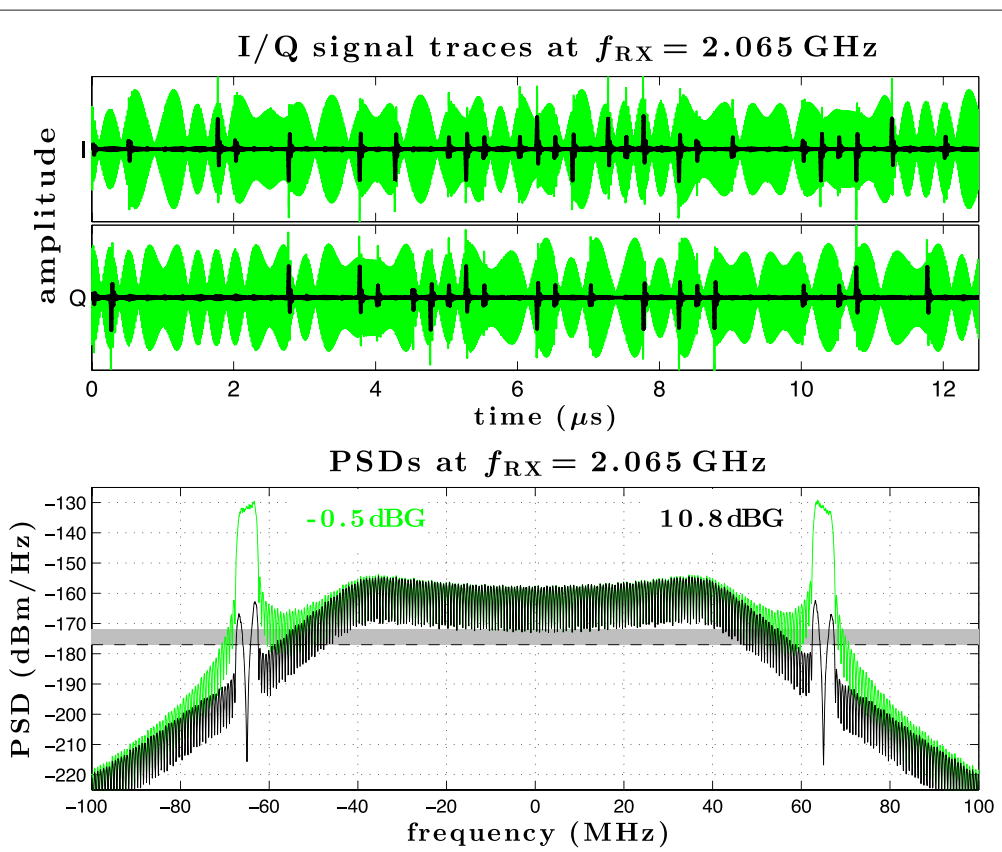

Figure 6 Quantification of receiver signal without thermal noise at wide bandwidth, with and without notch filter $(\Delta f=65 \mathrm{MHz})$. Upper panel: in-phase/quadrature (I/Q) signal traces for $f_{\mathrm{RX}}=2.065 \mathrm{GHz}$, for a $40-\mathrm{MHz}$ lowpass filter (green lines) and for a 40-MHz lowpass filter cascaded with a 65-MHz notch filter (black lines). Lower panel: power spectral densities and peakedness of the receiver signal for a 40-MHz lowpass filter (green) and for a 40-MHz lowpass filter cascaded with a 65-MHz notch filter (black). The thermal noise density is indicated by the horizontal dashed line. The width of the shaded band indicates the receiver noise figure $(5 \mathrm{~dB})$.

In Equation 5, the terms under the summation sign are functions of the nondimensionalized time $\bar{t}=\frac{2 \pi}{T} t$. Then, for a given transmitter power and the modulation pulse shape, if the discontinuities are due to the modulation pulse shape only and thus the time intervals between $t_{i}$ and $t_{i+1}$ are proportional to the unit interval $T$, the differences between $\bar{t}_{i}$ and $\bar{t}_{i+1}$ and thus the time average of the sum in Equation 5 are independent of the symbol rate. As a result, provided that the conditions for Equation 5 are met, for the given offset frequency $\Delta f$, transmitter power, and modulation pulse shape, the average interference power is proportional to the symbol rate raised to the power of $2 n$.

This is illustrated in Figure 7, where the upper panel shows the (highly oversampled) FIR root-raised-cosine filters ([15], for example) used for pulse shaping. All four filters have group delays equal to three times the unit interval $T$, two with roll-off factor one fourth (red and blue lines), and two with roll-off factor zero (black and green lines). The nonzero end values of the filters shown by the red and blue lines lead to discontinuities in the modulation signal $(n=1)$. The ratio of the unit intervals for these filters is equal to two (for the symbol rates 2 and $1 \mathrm{Mbit} / \mathrm{s}$, respectively), and thus, the ratio of the respective interference powers at high $\Delta f$ is $2^{2 n}=2^{2}$, or $6 \mathrm{~dB}$, as can be seen in the middle panel of Figure 7.
While for the filters shown by the black and green lines the modulating signal itself is continuous (the end values are zero), the first time derivative of the modulation signal is discontinuous $(n=2)$. The ratio of the unit intervals for these filters is equal to four (for the symbol rates 8 and $2 \mathrm{Mbit} / \mathrm{s}$, respectively), and thus, the ratio of the respective interference powers at high $\Delta f$ is $4^{2 n}=4^{4}$, or $24 \mathrm{~dB}$, as can be seen in the middle panel of Figure 7 . As can be seen in the lower panel of Figure 7, as the offset frequency $\Delta f$ increases, the impulsive component of the OOB interference becomes dominant, leading to a high peakedness of the interference.

As can also be seen from Equation 5, the average interference power depends on the impulse response $w(t)$ of the receiver lowpass filter and, for a filter of a given type and order, is proportional to its bandwidth. On the other hand, the thermal noise power is also proportional to the bandwidth of the receiver lowpass filter, and thus, the ratio of the powers of the interference and the thermal noise is independent of this bandwidth. This can be seen in the upper panels of Figure 4, where the increase in the bandwidth of the receiver lowpass filter from 5 to $40 \mathrm{MHz}$ (by $9 \mathrm{~dB}$ ) results in a 9-dB increase of both the interference and the thermal noise powers.

While the increase in the bandwidth does not affect the baseband PSD of either the interference or the thermal noise, widening of this bandwidth increases the 


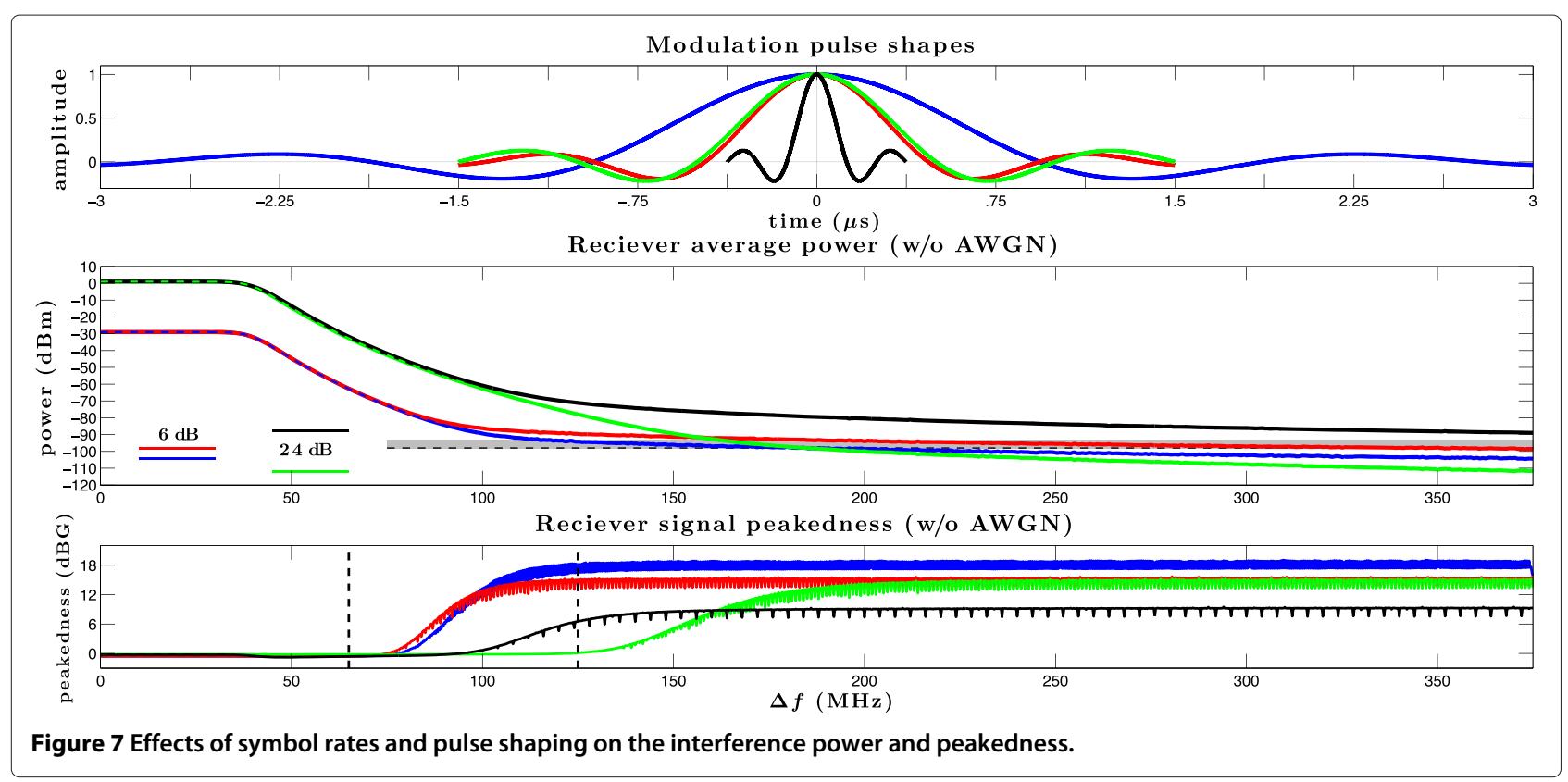

peakedness of the interference, enabling its more effective mitigation by the NDLs.

\subsection{Limitations of mainstream approaches to impulsive noise mitigation}

Since a signal of interest typically occupies a different and/or narrower frequency range than the noise, linear filters are typically applied to the incoming mixture of the signal and the noise in order to reduce the frequency range of the mixture to that of the signal. This reduces the power of the interference to a fraction of the total, limited to the frequency range of the signal. However, the noise having the same frequency power spectrum may have various peakedness and be impulsive (super-Gaussian) or nonimpulsive (sub-Gaussian). For example, white shot noise is much more impulsive than white thermal noise, while both have identically flat power spectra. Linear filtering cannot improve the signal-to-noise ratio (SNR) in a passband of interest, does not discriminate between impulsive and nonimpulsive noise contributions, and does not allow mitigation of the impulsive noise relative to the nonimpulsive. In addition, as can be justified by the central limit theorem [16], reduction in the bandwidth of an initially impulsive noise by linear filtering typically reduces the peakedness and makes the noise less impulsive (more 'Gaussian-like'), decreasing the ability to separate the signal from the noise based on the peakedness.

Effective suppression of impulsive interferences in the signal path typically requires nonlinear means, for example, digital processing based on order statistics, and various approaches to design of nonlinear receivers with improved performance in the presence of impulsive interference have been proposed. Many of these are model-based approaches, which rely on theoretical or empirical assumptions and models of interference distributions. For example, the $\alpha$-stable $[17,18]$ and Middleton class $A, B$, and $C[19,20]$ distributions are commonly used to model the interference in wireline [21] and wireless $[22,23]$ communications. Such approaches, designed under specific interference model assumptions, are often limited by parameter estimation schemes (e.g., are sensitive to inaccuracies in obtaining derivatives) and may not be robust under a model mismatch. Alternative methods that do not explicitly rely on noise distribution models have also been proposed. Those include receiver designs based on flexible classes of distributions (e.g., myriad filter [24-26], normal inverse Gaussian (NIG) [27]) or directly on the log-likelihood ratio shape (e.g., soft limiter [28], hole puncher [29], $p$-norm [27,30,31]).

While linear filters cannot increase the passband SNR, they can be optimal if the noise is purely Gaussian (e.g., thermal). Nonlinear filters, on the other hand, can improve quality of a signal if the latter is affected by a non-Gaussian interference ([32], for example). When the noise is Gaussian, however, nonlinear filters would be typically inferior to linear filters. For example, a median filter [33] can be significantly more effective than a linear averaging filter in suppression of impulsive noise but less effective in removal of Gaussian noise. In addition, nonlinear filters are generally not compatible with the existing linear systems as they introduce various types of nonlinear distortions to the signal of interest. As will be demonstrated later in this paper, one of the significant advantages of NDLs is that, while being nonlinear 
filters, their nonlinear behavior is intermittent and is controlled by a single parameter in a manner that makes them fully compatible with existing linear devices and systems.

Another key methodological distinction of NDLs is that they are deployed (in their either analog or digital implementations) sufficiently early in the signal chain to combine bandwidth reduction with interference mitigation. Numerical filtering algorithms (e.g., digital nonlinear filters) are typically deployed after the analog-to-digital conversion $(\mathrm{ADC})$, when the bandwidth of the signal + interference mixture is reduced to below half of the sampling rate and it is often already 'too late' to deal with non-Gaussian interference effectively. Indeed, the nonGaussian nature of interference can be viewed as a result of the 'coupling' of various interference components in a wide frequency band. Bandwidth reduction destroys this coupling, making the interference appear more 'Gaussianlike' and reducing our ability to distinguish it from the thermal noise and to effectively remove it. Thus, insufficient processing bandwidth often severely limits the effectiveness of state-of-art nonlinear interference mitigation techniques. While this can be overcome by increasing the sampling rate (and thus the acquisition bandwidth), this further exacerbates the memory and DSP intensity of numerical algorithms, making them unsuitable for real-time implementation and treatment of nonstationary noise.

\section{Nonlinear differential limiters}

In this section, we provide a brief introduction to NDLs. More comprehensive descriptions of NDLs, with detailed analysis and examples of various NDL configurations, nonadaptive as well as adaptive, can be found in [1-3].

\subsection{Theoretical foundation of NDLs}

For the optimal mitigation of non-Gaussian interference by nonlinear filters, it is imperative that the distributional properties of the interference are known, either a priori or through measurements. The 'blind' NDL-based approach proposed in this paper arises from the methodology introduced in [34], which relies on the transformation of discrete or continuous signals into normalized continuous scalar fields with the mathematical properties of distribution functions. This methodology enables a variety of nonlinear signal processing techniques that naturally incorporate the consideration of such distributional properties, including those which have no digital counterparts.

For example, as detailed in [34], the time-dependent amplitude distribution $\Phi(D, t)$ of a continuous signal $x(t)$ obtained in a time window $w(t)$ can be expressed as:

$$
\Phi(D, t)=w(t) * \mathcal{F}_{\Delta D}[D-x(t)]
$$

where $D$ is a threshold value, asterisk denotes convolution, and $\mathcal{F}_{\Delta D}(D)$ is a discriminator function that changes monotonically from zero to one in such a way that most of this change occurs over some characteristic range of threshold values $\Delta D$ around zero.

Since $\Phi(D, t)$ can be viewed as a surface in the threedimensional space $(t, D, \Phi)$, the expression:

$$
\Phi\left(D_{q}(t), t\right)=q, \quad 0<q<1
$$

defines $D_{q}(t)$ as a level (or contour) curve obtained from the intersection of the surface $\Phi=\Phi(D, t)$ with the plane $\Phi=q$, as illustrated in Figure 8 .

As is well known in differential geometry, an explicit (albeit differential) equation of the level curve $D_{q}(t)$ can be obtained by differentiating Equation 7 with respect to time (see, for example, [35], p. 551, equation (4.29)), leading to:

$$
\frac{\mathrm{d} D_{q}}{\mathrm{~d} t}=-\frac{\partial \Phi\left(D_{q}, t\right) / \partial t}{\phi\left(D_{q}, t\right)}+v\left[q-\Phi\left(D_{q}, t\right)\right], \quad v>0
$$

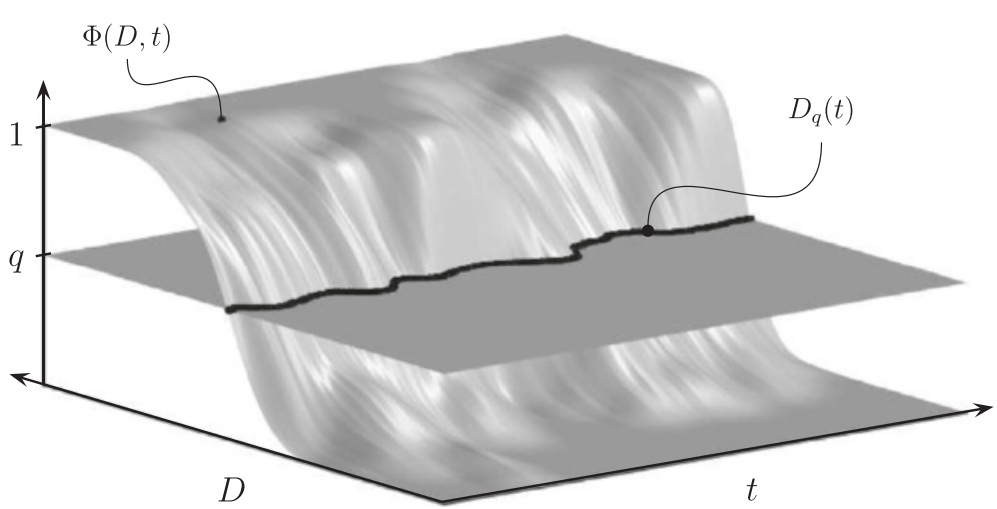

Figure $8 D_{q}(t)$ as a level curve of the distribution function $\Phi(D, t)$. 
In Equation 8, $\phi(D, t)=\partial \Phi(D, t) / \partial D$ is the amplitude density of $x(t)$ in the time window $w(t)$, and since $\Phi(D, t)$ is a monotonically increasing function of $D$ for all $t$, the added term in the right-hand side ensures the convergence of the solution to the chosen quantile order $q$ regardless of the initial condition.

It can be shown that, depending on the shape of the discriminator function $\mathcal{F}_{\Delta D}(D)$, Equation 8 corresponds to a variety of nonlinear filters with desired characteristics. For example, as demonstrated in our earlier work [34], in the limit $\Delta D \rightarrow 0$, Equation 8 describes an analog rank filter (e.g., a median filter for $q=1 / 2$ ) in an arbitrary time window $w(t)$, leading, as illustrated below, to the introduction of NDLs.

\subsection{First-order canonical differential limiter}

The digital median filter introduced in the early 1970s [33] is a widely recognized tool for removing outlier (i.e., impulsive) noise. In our prior work [34], we introduce analog rank filters in arbitrary time windows and derive differential and integro-differential equations that enable their implementation in analog feedback circuits. In particular, from equation (4.6) in [34], an expression for the output $\chi(t)$ of an 'exact' (or 'true') analog median filter in an exponential time window with the time constant $\tau_{0}$ can be written as:

$$
\dot{\chi}(t)=\lim _{\alpha \rightarrow 0} \frac{\frac{1}{2}-\mathcal{F}_{2 \alpha}[\chi(t)-\chi(t)]}{\int_{-\infty}^{t} \mathrm{~d} s \exp \left(\frac{s-t}{\tau_{0}}\right) f_{2 \alpha}[\chi(t)-\chi(s)]},
$$

where $x(t)$ is the input signal, $\mathcal{F}_{2 \alpha}(x)$ is a discriminator function with a characteristic width $2 \alpha$, and $f_{2 \alpha}(x)=\mathrm{d} \mathcal{F}_{2 \alpha}(x) / \mathrm{d} x$ is its respective probe. In Equation 9, $\mathcal{F}_{2 \alpha}(x)$ and $f_{2 \alpha}(x)$ are such that $\lim _{\alpha \rightarrow 0} \mathcal{F}_{2 \alpha}(x)=\theta(x)$ and $\lim _{\alpha \rightarrow 0} f_{2 \alpha}(x)=\delta(x)$, where $\theta(x)$ is the Heaviside unit step function [36] and $\delta(x)$ is the Dirac $\delta$-function [37]. In Equation 9, the parameter $\alpha$ can be called the resolution parameter.
Let us now choose a particularly simple discriminator function with a 'ramp' transition, such that the respective probe will be a boxcar function, as illustrated in the left-hand panel of Figure 9. In this case, since the main contribution to the integral in the denominator of Equation 9 will come from a relatively close proximity to the point $s=t$, for a finite and sufficiently large $\alpha$ such that $|\chi(t)-\chi(t)|$ generally remains smaller than the resolution parameter $\alpha$, except for relatively rare outliers with a typical duration much smaller than $\tau_{0}$, the denominator in Equation 9 can be approximated by a constant value equal to $\tau_{0} /(2 \alpha)$. Then, for a finite $\alpha$, Equation 9 becomes:

$$
\chi=x-\tau(|x-\chi|) \dot{\chi}
$$

where the time parameter $\tau=\tau(|x-\chi|)$ is given by:

$$
\tau(|x-\chi|)=\tau_{0} \times\left\{\begin{array}{cc}
1 & \text { for } \quad|x-\chi| \leq \alpha \\
\frac{|x-\chi|}{\alpha} & \text { otherwise }
\end{array}\right.
$$

as illustrated in the right-hand panel of Figure 9.

We shall call a filter described by Equations 10 and 11 a first-order canonical differential limiter $(C D L)$. Note that when the time parameter $\tau$ is a constant (e.g., in the limit $\alpha \rightarrow \infty)$, Equation 10 describes a first-order linear ana$\log$ filter ( $\mathrm{RC}$ integrator), wherein the rate of change of the output is proportional to the difference signal $x-\chi$. When the magnitude of the difference signal $|x-\chi|$ exceeds the resolution parameter $\alpha$, however, the rate of change of the output is proportional to the sign function of the difference signal and no longer depends on the magnitude of the incoming signal $\chi(t)$, providing an output insensitive to outliers with a characteristic amplitude determined by the resolution parameter.

\subsection{Higher order NDLs}

A high-order analog linear lowpass filter would be typically constructed as a first- (for odd-order filters) or
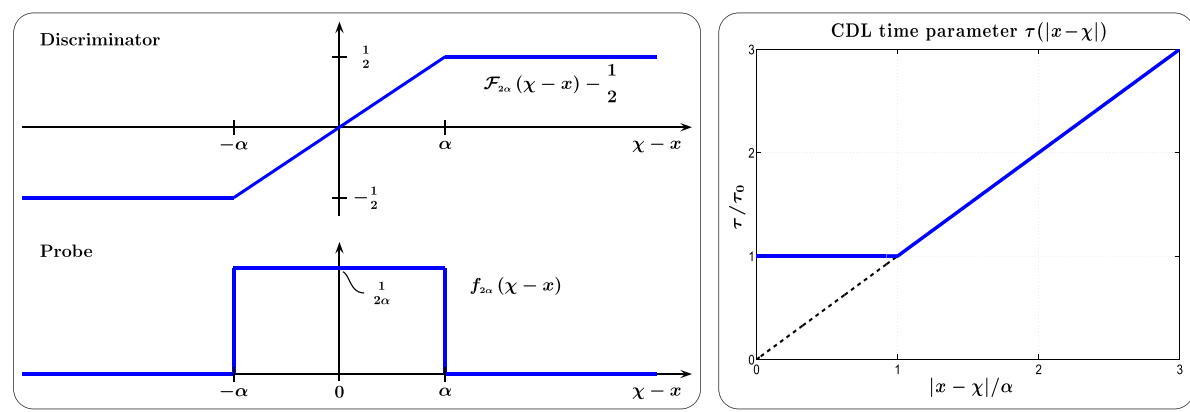

Figure 9 'Ramp' discriminator and CDL time parameter. Left panel: 'Ramp' discriminator and its respective boxcar probe. Right panel: CDL time parameter $\tau=\tau(|x-\chi|)$. 
second- (for even-order filters) order stage followed by cascaded second-order stages, typically arranged from the lowest to the highest quality factor. A similar approach can be taken to extend the previous example to higher order NDLs. For example, a third-order NDL can be constructed as a first-order CDL followed by a second-order linear filter, and a fourth-order NDL as a second-order NDL (introduced below) followed by a second-order linear filter. Such general constructions are of theoretical interest as it may be practically unnecessary to cascade the NDL stages. Indeed, the main burden of removing outliers will be carried out by the first stage, and the subsequent stages would be needed only to provide a desired frequency and phase response for the linear-regime NDL operation.

For even-order NDLs, a second-order NDL stage can be introduced as follows. Let us consider a second-order lowpass stage that can be described by the differential equation:

$$
\zeta(t)=z(t)-\tau \dot{\zeta}(t)-(\tau Q)^{2} \ddot{\zeta}(t)
$$

where $z(t)$ and $\zeta(t)$ are the input and the output signals, respectively (which can be real-, complex-, or vectorvalued), $\tau$ is the time parameter of the stage, $Q$ is the quality factor, and the dot and the double dot denote the first and the second time derivatives, respectively. Note that, when written in such a form, Equation 12 with $Q=0$ describes a first-order lowpass filter.

For a linear time-invariant filter, the time parameter $\tau$ and the quality factor $Q$ in Equation 12 are constants, so that, when the input signal $z(t)$ is increased by a factor of $K$, the output $\zeta(t)$ is also increased by the same factor, as is the difference between the input and the output. For convenience, we will call the difference between the input and the output $z(t)-\zeta(t)$ the difference signal. A transient outlier in the input signal would result in a transient outlier in the difference signal of a filter, and an increase in the input outlier by a factor of $K$ would result, for a linear filter, in the same factor increase in the respective outlier of the difference signal. If a significant portion of the frequency content of the input outlier is within the passband of the linear filter, the output will typically also contain an outlier corresponding to the input outlier, and the amplitudes of the input and the output outliers will be proportional to each other. A reduction (limiting) of the output outliers, while preserving the relationship between the input and the output for the portions of the signal not containing the outliers, can be achieved by proper dynamic modification of the filter parameters $\tau$ and $Q$ in Equation 12 based on the magnitude (for example, the absolute value) of the difference signal. A filter comprising such dynamic modification of the filter parameters based on the magnitude of the difference signal will be called an NDL.

Since at least one of the filter parameters depends on the instantaneous magnitude of the difference signal, the differential equation describing such a filter is nonlinear. However, even though in general an NDL is a nonlinear filter, if the parameters remain constant as long as the magnitude of the difference signal remains within a certain range, the behavior of the NDL will be linear during that time. Thus, an NDL can be configured to behave linearly as long as the input signal does not contain outliers. By specifying a proper dependence of the NDL filter parameters on the difference signal, it can be ensured that, when the outliers are encountered, the nonlinear response of the NDL limits the magnitude of the respective outliers in the output signal.

A comprehensive discussion and illustrative examples of various dependencies of the NDL parameters on the difference signal can be found in [1-3]. For example, one can set the quality factor in Equation 12 to a constant value and allow the time parameter $\tau$ to be a nondecreasing function of the absolute value of the difference signal satisfying the following equation:

$$
\tau(|z-\zeta|)=\tau_{0} \times\left\{\begin{array}{cc}
1 & \text { for }|z-\zeta| \leq \alpha \\
>1 & \text { otherwise }
\end{array}\right.
$$

where $\alpha>0$ is the resolution parameter. A particular example can be given by

$$
\tau(|z-\zeta|)=\tau_{0} \times\left\{\begin{array}{cc}
1 & \text { for } \quad|z-\zeta| \leq \alpha \\
\left(\frac{|z-\zeta|}{\alpha}\right)^{\beta} & \text { otherwise }
\end{array}\right.
$$

with $\beta>0$. Parameter $\beta$ in Equation 14 controls the behavior of the NDL in the presence of outliers - the larger its value, the stronger the suppression of outliers. From practical considerations, the value $\beta=1$ is convenient, so we refer to the NDL with $\beta=1$ as a CDL. If stronger suppression is desirable, then we can use $\beta>1$, which we call a differential over-limiter (DoL).

It should be easily seen from Equations 13 or 14 that in the limit of a large resolution parameter, $\alpha \rightarrow \infty$, an NDL becomes equivalent to the respective linear filter with $\tau=\tau_{0}=$ const. This is an important property of the proposed NDL, enabling its full compatibility with linear systems. At the same time, when the noise affecting the signal of interest contains impulsive outliers, the signal quality (e.g., as characterized by a SNR, a throughput capacity of a communication channel, or other measures of signal quality) exhibits a global maximum at a certain finite value of the resolution parameter $\alpha=\alpha_{0}$. As illustrated in the next section, this property of an NDL 
enables its use for improving the signal quality in excess of that achievable by the respective linear filter, effectively reducing the spectral density of the interference in the signal passband without significantly affecting the signal of interest.

\section{Examples of mitigation of out-of-band interference by NDLs}

The incoming 'native' (in-band) RX signal used in the examples of Figures 10, 11, 12, and 13 was a QPSK signal with the I/Q modulating signals as two independent random bit sequences with the rate $4.8 \mathrm{Mbit} / \mathrm{s}$. An FIR root-raised-cosine (RRC) filter with a roll-off factor of one fourth and group delay $3 T$ was used for the RX incoming signal pulse shaping, and the same FIR filter was used for matched filtering in the baseband. In all examples, the signal-to-noise ratio for the RX signal was measured in the baseband, after applying the matched FIR filter. The PSD of the RX signal without noise was approximately $-167 \mathrm{dBm} / \mathrm{Hz}$ in the baseband, leading to the $\mathrm{S} / \mathrm{N}$ ratio without interference of approximately $5 \mathrm{~dB}$, as indicated by the upper horizontal dashed lines in Figures 10 and 12 . The OOB interference was created by the TX signal used in the examples of Section 2. In all the examples of this section, the NDLs were fourth-order 'Butterworthlike' filters constructed as a second-order constant-Q CDL with the pole quality factor $Q=1 / \sqrt{2+\sqrt{2}}$ and the initial cutoff frequency $f_{0}=5.25 \mathrm{MHz}$, followed by a second-order linear lowpass filter with $Q=1 / \sqrt{2-\sqrt{2}}$ and the same cutoff frequency.

In Figures 10 and 11, the receiver with the 40-MHz lowpass filter was tuned to $f_{\mathrm{RX}}=2.125 \mathrm{GHz}$. Figure 10 shows the SNR in the receiver baseband as a function of the NDL resolution parameter $\alpha$. In the limit of a large-resolution parameter, an NDL is equivalent to the respective linear filter (in this example, the fourth-order Butterworth lowpass filter with the cutoff frequency $f_{0}=5.25 \mathrm{MHz}$ ), resulting in the same signal quality of the filtered output as provided by the linear filter (indicated by the lower horizontal dashed line). When viewed as a function of the resolution parameter, however, the signal quality of the NDL output exhibits a global maximum at some $\alpha=\alpha_{0}$. This property of an NDL enables its use for improving the signal quality in excess of that achievable by the respective linear filter, effectively reducing the in-band impulsive interference. As can be seen in Figure 10, when linear processing is used, the OOB interference reduces the SNR by approximately $6.7 \mathrm{~dB}$. The NDL with $\alpha=\alpha_{0}$ improves the SNR by approximately $4.8 \mathrm{~dB}$, suppressing the $\mathrm{OOB}$ interference by approximately a factor of three.

For the resolution parameter of the NDL set to $\alpha=\alpha_{0}$, Figure 11 shows the time domain I/Q traces and PSDs of
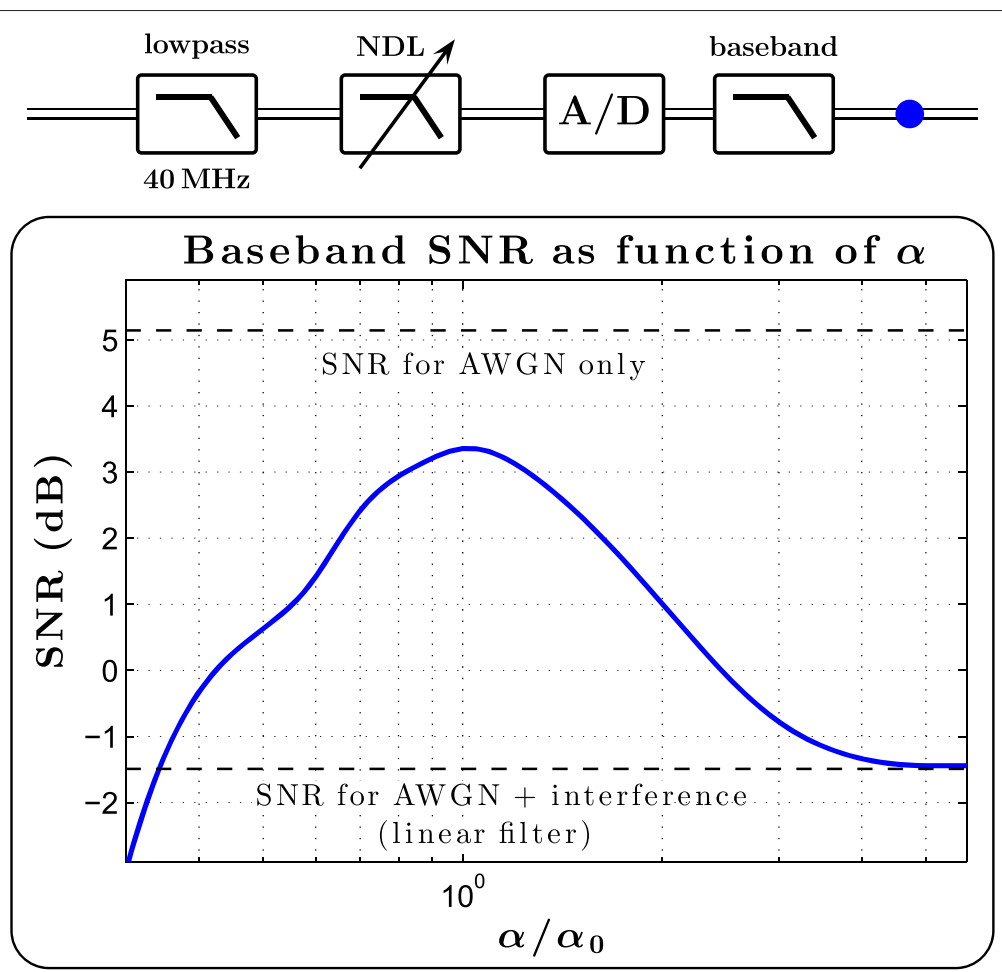

Figure 10 SNR in the receiver baseband as a function of the NDL resolution parameter $\boldsymbol{\alpha}$. The RX frequency is $f_{\mathrm{RX}}=2.125 \mathrm{GHz}$, and the NDL follows the 40-MHz lowpass filter. 


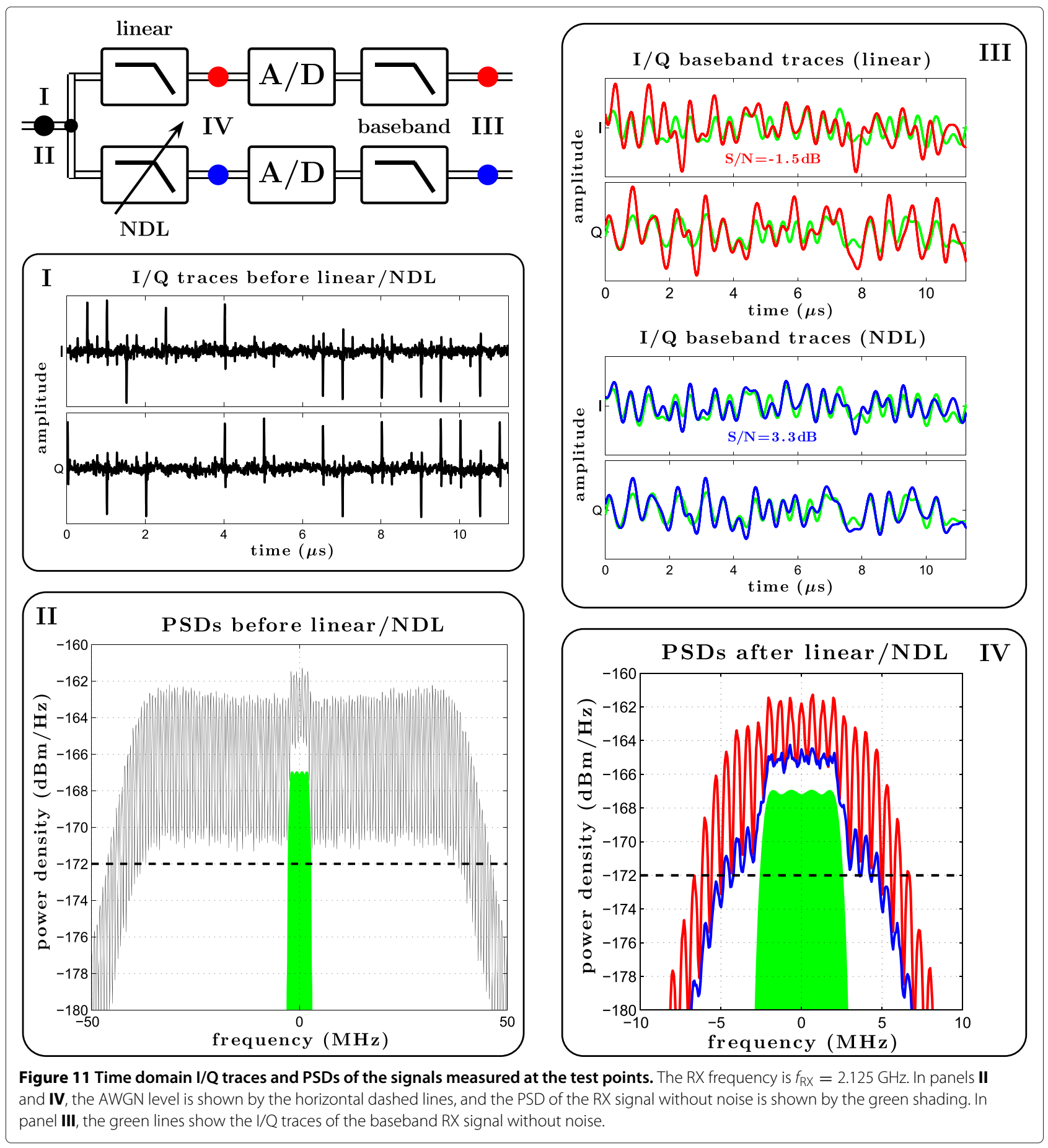

the signals measured at the test points indicated by the fat colored dots on the signal path diagram outlined in the upper left of the figure. In panels II and IV, the AWGN level is shown by the horizontal dashed lines, and the PSD of the RX signal without noise is shown by the green shading. In panel III, the green lines show the I/Q traces of the baseband RX signal without noise. In panel IV of Figure 11, the fact that the NDL indeed reduces the spectral density of the interference without significantly affecting the signal of interest can be deduced and gauged from observing how the quasiperiodic structure of the PSD is affected by the NDL in comparison with the linear filter.

If the Shannon formula [38] is used to calculate the capacity of a communication channel, the baseband SNR increase from -1.5 to $3.3 \mathrm{~dB}$ provided by the NDL in 

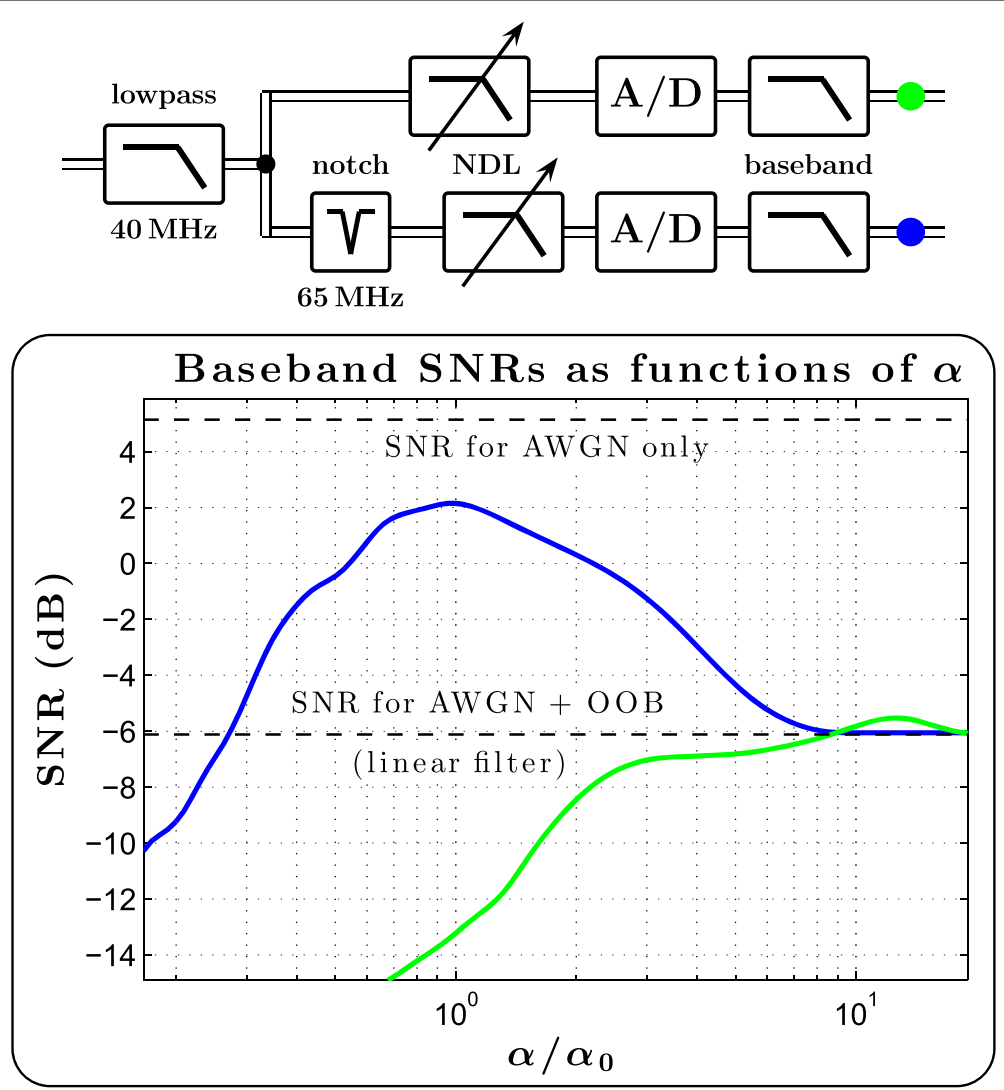

Figure 12 SNRs in the receiver baseband as functions of the NDL resolution parameter $\boldsymbol{\alpha}$. The RX frequency is $f_{\mathrm{RX}}=2.065 \mathrm{GHz}$. Green line: the $\mathrm{NDL}$ is applied directly to the output of the 40-MHz lowpass filter. Blue line: a $65-\mathrm{MHz}$ notch filter precedes the NDL.

the examples of Figures 10 and 11 results in a $114 \%$ (2.14 times) increase in the channel capacity.

Figure 12 shows the SNRs in the receiver baseband as functions of the NDL resolution parameter $\alpha$ for the $\mathrm{RX}$ frequency $f_{\mathrm{RX}}=2.065 \mathrm{GHz}$, when the NDL is applied directly to the output of the 40-MHz lowpass filter (green line) and when a $65-\mathrm{MHz}$ notch filter precedes the NDL (blue line). As can be seen in Figure 12 from the distance between the horizontal dashed lines, when linear processing is used, the OOB interference reduces the SNR by approximately $11 \mathrm{~dB}$.

As was discussed in Section 2 (see, in particular, the description of Figure 6), the response of the receiver 40$\mathrm{MHz}$ lowpass filter at $65 \mathrm{MHz}$ is relatively large, and the contribution of the TX signal in its nominal band (part I of the interference in Figure 2) into the total interference is significant, which makes the total interference sub-Gaussian ( $-0.5-\mathrm{dBG}$ peakedness). Thus, an NDL deployed immediately after the 40-MHz lowpass filter will not be effective in suppressing the interference, as can be seen from the SNR curve shown by the green line in Figure 12 . However, a $65-\mathrm{MHz}$ notch filter preceding the NDL attenuates the nonimpulsive part of the interference without affecting either the signal of interest or the PSD of the impulsive interference, making the interference impulsive and enabling its effective mitigation by the subsequent NDL. This can be seen from the SNR curve shown by the blue line in Figure 12, where the NDL with $\alpha=\alpha_{0}$ improves the SNR by approximately $8.2 \mathrm{~dB}$, suppressing the OOB interference by approximately a factor of 6.6.

For the resolution parameter of the NDL set to $\alpha=\alpha_{0}$, Figure 13 shows the time domain I/Q traces and the PSDs of the signals measured at the test points indicated by the fat colored dots on the signal path diagram in the upper left of the figure. The RX frequency is $f_{\mathrm{RX}}=2.065 \mathrm{GHz}$, and the notch is at $65 \mathrm{MHz}$. In panels II and IV, the AWGN level is shown by the horizontal dashed lines, and the PSD of the RX signal without noise is shown by the green shading. In panel III, the green lines show the I/Q traces of the baseband RX signal without noise. In panel IV of Figure 13, the fact that the NDL indeed reduces the spectral density of the interference without significantly affecting the signal of interest can be deduced and gauged from observing how the quasiperiodic structure of the PSD is affected by the NDL in comparison with the linear filter. 


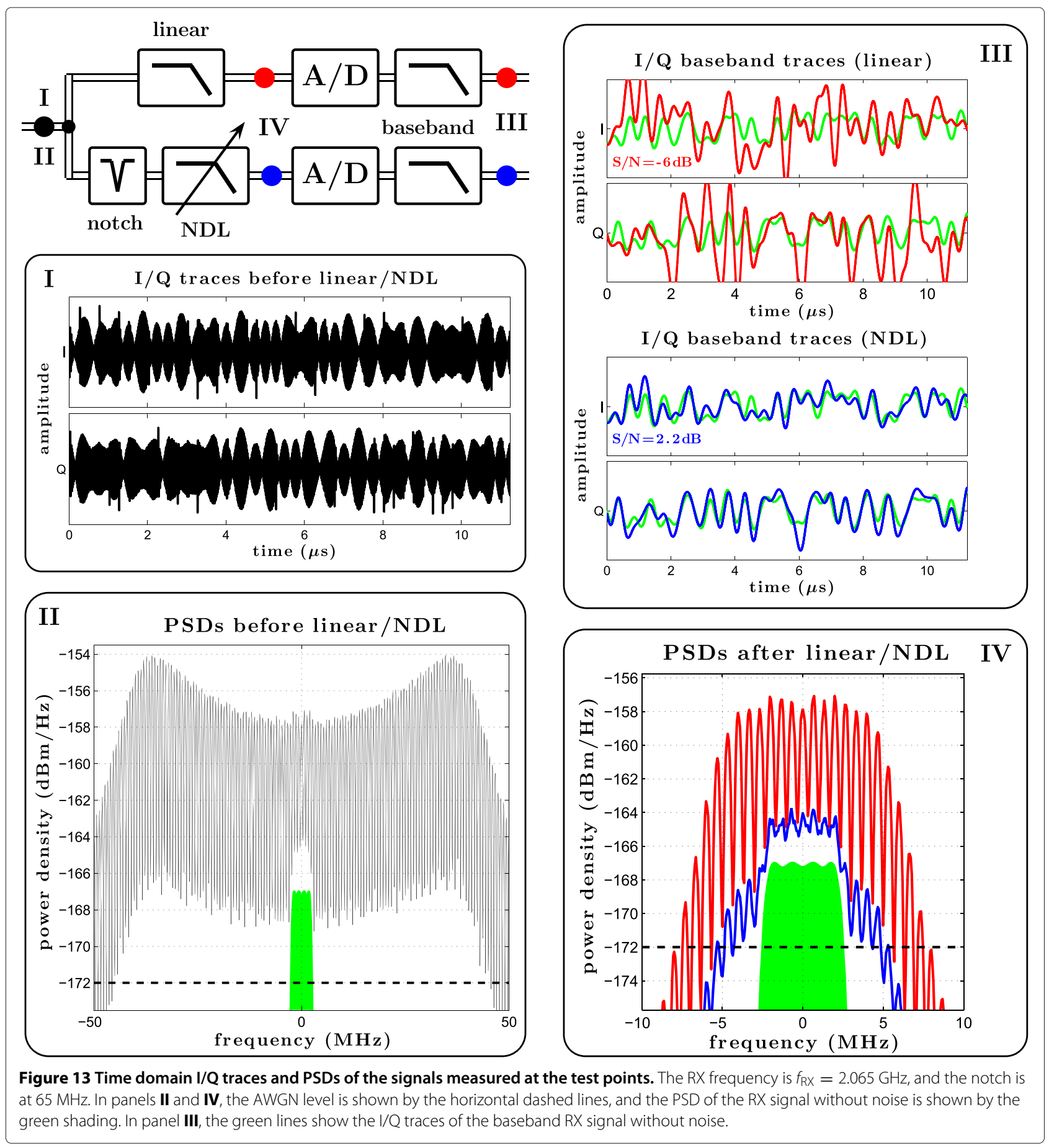

If the Shannon formula [38] is used to calculate the capacity of a communication channel, the baseband SNR increase from -6 to $2.2 \mathrm{~dB}$ provided by the NDL in the examples of Figures 12 and 13 results in a $337 \%$ (4.37 times) increase in the channel capacity.

The value of $\alpha_{0}$ that maximizes the signal quality may vary in a wide range depending on the composition of the signal + noise mixture, for example, on the SNR and the relative spectral and temporal structures of the signal and the noise. ANDL configurations (see [1-3]) contain a subcircuit (characterized by a gain parameter) that monitors a chosen measure of the signal + noise mixture and provides a time-dependent resolution parameter $\alpha=\alpha(t)$ to the main NDL circuit, making it suitable for improving the quality of nonstationary signals under time-varying noise conditions. 


\section{Conclusions}

Interference from various technogenic (man-made) sources, unintentional as well as intentional, typically has temporal and/or amplitude structure, and its amplitude distribution is usually non-Gaussian. A simplified explanation of the non-Gaussian (and often impulsive) nature of a technogenic noise produced by digital electronics and communication systems can be as follows. An idealized discrete-level (digital) signal can be viewed as a linear combination of Heaviside unit step functions ([36], for example). Since the derivative of the Heaviside unit step function is the Dirac $\delta$-function ([37], for example), the derivative of an idealized digital signal is a linear combination of Dirac $\delta$-functions, which is a limitlessly impulsive signal with zero interquartile range and infinite peakedness. The derivative of a 'real' (i.e., no longer idealized) digital signal can thus be viewed as a convolution of a linear combination of Dirac $\delta$-functions with a continuous kernel. If the kernel is sufficiently narrow (for example, the bandwidth is sufficiently large), the resulting signal will appear as an impulse train protruding from a continuous background signal. Thus, impulsive interference occurs naturally in digital electronics as " $\mathrm{d} i / \mathrm{d} t$ " (inductive) noise or as the result of the coupling (for example, capacitive) between various circuit components and traces, leading to the so-called 'platform noise' ([39], for example).

In this paper, we focus on particular illustrative mechanisms of impulsive interference in digital communication systems resulting from the nonsmooth nature of any physically realizable modulation scheme for transmission of a digital (discontinuous) message. Even modulation schemes designed to be 'smooth', e.g., continuous-phase modulation, are, in fact, not smooth because their higher order time derivatives still contain discontinuities.

The non-Gaussian nature of technogenic interference provides an opportunity for its mitigation by nonlinear filtering that is more effective than the mitigation achievable by linear filters. When a linear filter is used to suppress interference outside the passband of interest, the filtered signal quality is not influenced by the type of the amplitude distribution of the interfering signal, as long as the total power and the spectral composition of the interference is the same. It may be possible to reduce the spectral density of the in-band technogenic interference (that is, in the signal's passband) without significantly affecting the signal of interest by introducing an appropriately chosen feedback-based nonlinearity into the response of a filter. As a result, the signal quality can be improved in excess of that achievable by the respective linear filter.

In this paper, we describe such nonlinear filters (NDLs), outline a methodology for mitigation of technogenic interference in communication channels by NDLs, and provide several examples of such mitigation. We demonstrate that an NDL replacing a linear filter in the receiver channel can improve the receiver by increasing the signal quality in the presence of man-made noise and thus the capacity of a communication channel.

\subsection{Comment on use methodology}

As was stated in our prior work [1-3] and illustrated in Section 2 of this paper, the distributions of non-Gaussian signals are generally modifiable by linear filtering, and non-Gaussian interference can often be converted from sub-Gaussian into super-Gaussian, and vice versa, by linear filtering that does not affect the signal of interest. As a result, employing appropriate linear filtering preceding an NDL in a signal chain can greatly improve effectiveness of NDL-based interference mitigation. While we have previously outlined several approaches to such distribution modification by linear front end (LFE) filtering, and to identifying non-Gaussian components in an interfering signal $[1,2]$, the development of systematic procedures for identification of non-Gaussian interference components and for design of appropriate LFE filtering remains a challenging task that is a subject of ongoing research.

\subsection{Comment on digital NDLs}

Conceptually, NDLs are analog filters that combine bandwidth reduction with mitigation of interference. For the interference to appear strongly impulsive, the bandwidth of the receiver lowpass filter needs to be much larger than a typical value of $\left(t_{i+1}-t_{i}\right)^{-1}$ in Equation 2, and effective use of an NDL may require that its input signal has a bandwidth much larger than the bandwidth of the RX signal of interest. Thus, the best conceptual placement for an NDL is in the analog part of the signal chain, for example, as part of the antialiasing filter preceding an ADC, as shown in panel (a) of Figure 14. However, digital NDL implementations may offer many advantages typically associated with digital processing, including simplified development and testing, configurability, and reproducibility.

While near-real-time finite-difference implementations of the NDLs described in Section 3 would be relatively simple and computationally inexpensive, their use would still require a digital signal with a sampling rate much higher than the Nyquist rate of the signal of interest. Increasing the sampling rate of a high-resolution converter in order to enable the use of an NDL would be impractical for many reasons, including the ADC cost and its saturation by high-amplitude impulsive outliers. Instead, as illustrated in panel (b) of Figure 14, a low-bit high-rate A/D converter should be used to provide the input to a digital NDL. Then, the NDL output can be 


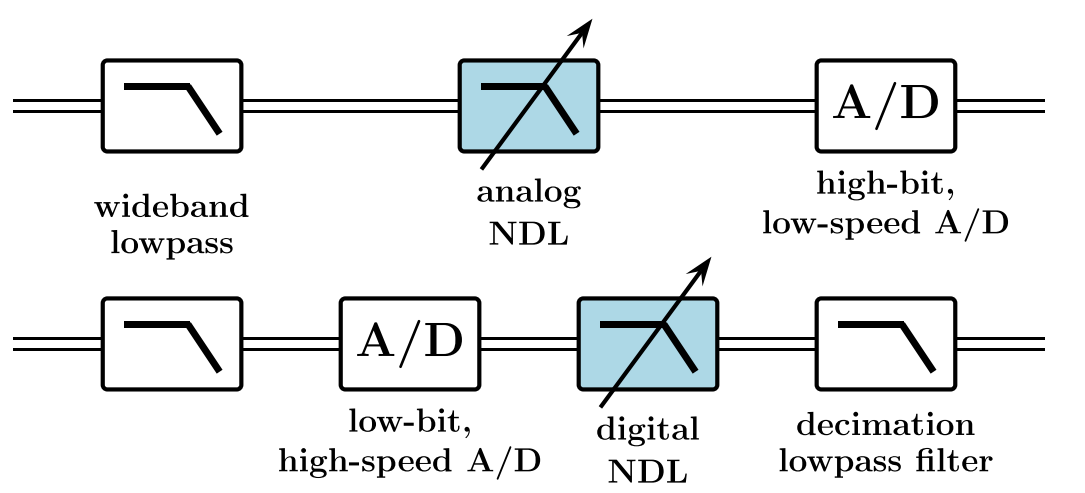

(a)

Figure 14 Analog (panel (a)) and digital (panel (b)) NDL deployment.

downsampled (after appropriate digital lowpass filtering) to provide the desired high-resolution signal at a lower sampling rate.

\section{Endnotes}

${ }^{a}$ For example, the out-of-band (OOB) emissions of a transmitter may be greatly reduced by employing a high-quality bandpass filter in the antenna circuit of the transmitter. Such an additional filter, however, may negatively affect other properties of a system, for example, by increasing its cost and power consumption (due to the insertion loss of the filter).

${ }^{\mathrm{b}}$ One will encounter discontinuities in a derivative of some order in the modulating signal sooner or later, since any physical pulse shaping is implemented using causal filters of finite order.

${ }^{\mathrm{c}}$ In general, if $n$ is the order of a causal analog filter, then $n-1$ is the order of the first discontinuous derivative of its impulse response.

${ }^{\mathrm{d}}$ Equation (2) will still accurately represent the instantaneous power in the quadrature receiver if the 'real' (physical) modulating signal can be expressed as $A(t)=\psi(t) * A_{T}(t)$, where the convolution kernel $\psi(t)$ is a lowpass filter of a bandwidth much larger than $|\Delta f|$.

\section{Appendix}

\section{A Simulation parameters}

The transmitter signal used in all simulations was a QPSK signal with the I/Q modulating signals as two independent random bit sequences. In all simulations except those shown in Figure 7, the symbol rate was $4 \mathrm{Mbit} / \mathrm{s}$ (unit interval $T=250 \mathrm{~ns}$ ), and an FIR RRC filter ([15], for example) with the roll-off factor one fourth and the group delay $3 T$ was used for pulse shaping. The average TX signal power in all simulations was set to $125 \mathrm{~mW}(21 \mathrm{dBm})$, and it was assumed that the additional path/coupling loss at any RX frequency was $50 \mathrm{~dB}$, except for the TX signals shaped with the filters shown by black and green lines in Figure 7, where it was $20 \mathrm{~dB}$.

A rather small transmission power $(6 \mathrm{~dB}$ below a typical cellular phone power of $27 \mathrm{dBm}$ ) and a relatively large $50-\mathrm{dB}$ loss were chosen as somewhat of a 'safety margin' to ensure that, even if the $\mathrm{OOB}$ emissions are significantly (e.g., by 20 to $30 \mathrm{~dB}$ ) reduced by a carefully selected combination of the roll-off factor and the group delay of the shaping filter, and/or by reducing the bandwidth (symbol rate) of the TX signal, the spectral density of the interference may still be comparable with or dominate over the spectral density of the thermal noise for a respectively smaller path/coupling loss and/or larger transmit power.

The quasiperiodic time domain structures of the spectrograms in Figure 3, and of the time domain traces seen in the upper panels of Figures 5 and 6, and in panel I of Figure 11, are related to the unit interval $T$ and have a period $T=250 \mathrm{~ns}$. The quasiperiodic structures that can be seen in the PSDs shown in the lower panels of Figures 5 and 6, and in panels II and IV of Figures 11 and 13, are related to the group delay of the FIR pulse shaping filters and have a period equal to half of the inverse group delay or $(6 T)^{-1}=2 / 3 \mathrm{MHz}$.

As can be seen in the lower panels of Figure 4, the peakedness of the interference also exhibits a quasiperiodic structure (quasiperiodic local minima). This period is related to the symbol rate $T^{-1}$ as $(2 T)^{-1}=2 \mathrm{MHz}$ or half of the symbol rate. In those simulations, the $f_{\mathrm{RX}}$ sampling interval of $1.25 \mathrm{MHz}$ was used. This is why only one out of four local minima is visible, and the apparent period of peakedness in Figure 4 is $10 \mathrm{MHz}$. The local minima in the peakedness plots shown in the lower panel of Figure 7 also occur at halves of the respective symbol rates. For example, the peakedness shown by the black line has a structure with the period $4 \mathrm{MHz}$.

A constant $5-\mathrm{dB}$ noise figure of the receiver was assumed at all receiver frequencies $f_{\mathrm{RX}}$. This, combined 
with the $-177-\mathrm{dBm} / \mathrm{Hz}$ two-sided PSD of the thermal noise at room temperature, leads to the total AWGN noise level of $-172 \mathrm{dBm} / \mathrm{Hz}$. The incoming $\mathrm{RX}$ signal used in Figures $10,11,12$, and 13 was a QPSK signal with the I/Q modulating signals as two independent random bit sequences with the rate of $4.8 \mathrm{Mbit} / \mathrm{s}$. An FIR RRC filter with roll-off factor one fourth and group delay $3 T$ was used for the RX incoming signal pulse shaping, and the same FRI filter was used for the matched filtering in the baseband. The PSD of the RX signal without noise was approximately $-167 \mathrm{dBm} / \mathrm{Hz}$ in the baseband, leading to the $\mathrm{S} / \mathrm{N}$ ratio without interference of approximately $5 \mathrm{~dB}$.

\begin{abstract}
Abbreviations
$\mathrm{ACl}$, adjacent-channel interference; ADC, analog-to-digital converter; AMC, adaptive modulation and coding; $A N D L$, adaptive nonlinear differential limiter; AWGN, additive white Gaussian noise; A/D, analog-to-digital; dBm, dB per milliwatt; DoL, differential over-limiter; DSP, digital signal processing; EMC, electromagnetic compatibility; EMI, electromagnetic interference; FIR, finite impulse response; IMD, intermodulation distortion; I/Q, in-phase/quadrature; LFE, linear front end; MANET, mobile ad hoc network; Mbit/s, megabit per second; NDL, nonlinear differential limiter; OOB, out-of-band; PSD, power spectral density; QAM, quadrature amplitude modulation; QPSK, quadrature phase-shift keying (4-QAM); RBW, resolution bandwidth (electronic signal term used in spectrum analyzers and EMI/EMC testing); RF, radio frequency; RRC, root-raised-cosine; $\mathrm{RX}$, receiver; SDR, software-defined radio; SNR, signal-to-noise ratio; S/N, signal-to-noise; TX, transmitter.
\end{abstract}

\section{Competing interests}

This work was supported in part by the National Science Foundation under Grant Number 1314790. The authors have no other relationships or activities that could appear to have influenced the submitted work.

\section{Acknowledgements}

The authors would like to thank Malcolm D Macleod of QinetiQ and Bala Natarajan of Kansas State University for their valuable suggestions and critical comments.

\begin{abstract}
Author details
${ }^{1}$ Avatekh Inc., 901 Kentucky Street, Suite 303, Lawrence, KS 66044, USA. ${ }^{2}$ Dept. of Electrical and Computer Engineering, Kansas State University, Manhattan, KS 66506, USA. ${ }^{3}$ Dept. of Mathematics, University of Leicester, Leicester LE1 7RH, UK. ${ }^{4}$ BAE Systems Technology Solutions, 6 New England Executive Park, Burlington, MA 01803, USA.
\end{abstract}

Received: 17 August 2014 Accepted: 29 January 2015

Published online: 15 February 2015

\section{References}

1. AV Nikitin, Method and Apparatus for Signal Filtering and for Improving Properties of Electronic Devices. US patent 8,489,666 (2013)

2. Nikitin, AV, Method and Apparatus for Signal Filtering and for Improving Properties of Electronic Devices. US Patent Application Publications 2013/0297665 (Nov. 7, 2013), 2013/0339418 (Dec. 19, 2013), and 2014/0195577 (July 10, 2014)

3. AV Nikitin, RL Davidchack, TJ Sobering, in Proceedings of IEEE Military Communications Conference 2013. Adaptive Analog Nonlinear Algorithms and Circuits for Improving Signal Quality in the Presence of Technogenic Interference (San Diego, CA) p. 2013

4. AV Nikitin, RL Davidchack, JE Smith, in Proceedings of 3rd IMA Conference on Mathematics in Defence. Out-of-band and adjacent-channel interference reduction by analog nonlinear filters (Malvern, UK) p. 2013

5. EM Royer, CK Toh, A review of current routing protocols for ad-hoc mobile wireless networks. IEEE Pers. Commun. 6(2), 46-55 (1999)
6. SWeber, JG Andrews, N Jindal, The Effect of Fading, Channel Inversion, and Threshold Scheduling on Ad Hoc Networks. IEEE Trans. Inf. Theory. 53(11), 4127-4149 (2007)

7. H Inaltekin, M Chiang, HV Poor, SB Wicker, On Unbounded Path-Loss Models: Effects of Singularity on Wireless Network Performance. IEEE J. Selected Areas Commun. 27(7), 1078-1092 (2009)

8. S Basagni, M Conti, S Giordano, (eds.) Stojmenovic I, Mobile Ad Hoc Networking. (Wiley-IEEE Press, New York, 2004)

9. AV Nikitin, in Proc. IEEE Radio and Wireless Symposium. On the Impulsive Nature of Interchannel Interference in Digital Communication Systems (Phoenix, AZ, 2011), pp. 118-121

10. AV Nikitin, On the interchannel interference in digital communication systems, its impulsive nature, and its mitigation. EURASIP J Adv. Signal Process. 2011(137) (2011)

11. AV Nikitin, M Epard, JB Lancaster, RL Lutes, EA Shumaker, Impulsive interference in communication channels and its mitigation by SPART and other nonlinear filters. EURASIP J. Adv. Signal Process. 2012(79) (2012)

12. L Cohen, Time-frequency analysis. (Prentice-Hall, Englewood, NJ, 1995)

13. M Abramowitz, IA Stegun (eds.), Handbook of Mathematical Functions with Formulas, Graphs, and Mathematical Tables, 9th printing, (Dover, New York, 1972)

14. A Hyvärinen, J Karhunen, E Oja. Independent component analysis (Wiley, New York, 2001)

15. JG Proakis, DG Manolakis, Digital signal processing: principles, algorithms, and applications, 4th edn. (Prentice Hall, 2006)

16. R Durrett, Probability: Theory and examples, 4th edn. (Cambridge University Press, 2010)

17. G Samorodnitsky, MS Taqqu, Stable Non-Gaussian Random Processes: Stochastic Models with Infinite Variance. (Chapman and Hall, New York, 1994)

18. GA Tsihrintzis, CL Nikias, Fast estimation of the parameters of alpha-stable impulsive interference. IEEE Trans. Signal Process. 44(6), 1492-1503 (1996)

19. D Middleton, Statistical-Physical Models of Electromagnetic Interference. IEEE Trans. Electromagnetic Compatibility. EMC-19(3), 106-127 (1977)

20. D Middleton, Non-Gaussian noise models in signal processing for telecommunications: New methods and results for class A and class B noise models. IEEE Trans. Inf. Theory. 45(4), 1129-1149 (1999)

21. M Zimmermann, K Dostert, Analysis and modeling of impulsive noise in broad-band powerline communications. IEEE Trans. Electromagnetic Capability. 44, 249-258 (2002)

22. X Yang, AP Petropulu, Co-channel interference modeling and analysis in a Poisson field of interferers in wireless communications. IEEE Trans. Signal Process. 51, 64-76 (2003)

23. K Gulati, BL Evans, JG Andrews, KR Tinsley, Statistics of co-channel interference in a field of Poisson and Poisson-Poisson clustered interferers. IEEE Trans. Signal Proc. 58(12), 6207-6222 (2010)

24. JG Gonzalez, GR Arce, Optimality of the myriad filter in practical impulsive-noise environments. IEEE Trans. Signal Process. 49(2), 438-441 (2001)

25. P Zurbach, JG Gonzalez, GR Arce, Weighted myriad filters for image processing. IEEE Int Symp. Circuits Syst. 2, 726-729 (1996)

26. JG Gonzalez, GR Arce, Statistically-efficient filtering in impulsive environments: weighted myriad filters. EURASIP J. Appl. Signal Process. 2002, 4-20 (2002)

27. W Gu, G Peters, L Clavier, F Septier, I Nevat, in 2012 International Symposium on Wireless Communication Systems (ISWCS). Receiver study for cooperative communications in convolved additive $\mathrm{E} \pm$-stable interference plus Gaussian thermal noise, (2012), pp. 451-455

28. GL Stuber, Soft-limiter receivers for coded DS/DPSK systems. IEEE Trans. Commun. 38, 46-53 (1990)

29. S Ambike, J llow, D Hatzinakos, Detection for binary transmission in a mixture of Gaussian noise and impulsive noise modeled as an alpha-stable process. IEEE Signal Process. Lett. 1(3), 55-57 (1994)

30. NC Beaulieu, H Shao, J Fiorina, P-order metric UWB receiver structures with superior performance. IEEE Trans. Commun. 56(10), 1666-1676 (2008)

31. W Gu, L Clavier, Decoding metric study for turbo codes in very impulsive environment. IEEE Commun. Lett. 16(2), 256-258 (2012)

32. HV Poor, An Introduction to signal detection and estimation. (Springer, 1998)

33. JW Tukey, Exploratory Data Analysis. (Addison-Wesley, 1977)

34. AV Nikitin, RL Davidchack, Signal analysis through analog representation.Proc. R. Soc. Lond. A. 459(2033), 1171-1192 (2003) 
35. IN Bronshtein, KA Semendiaev, Handbook of Mathematics, 3rd edn. (Springer, 1997)

36. R Bracewell, The Fourier Transform and Its Applications, 2000 chap. "Heaviside's Unit Step Function, H(x)", 3rd edn. (McGraw-Hill, New York) pp. 61-65

37. PAM Dirac, The Principles of Quantum Mechanics, 4th edn. (Oxford University Press, London, 1958)

38. CE Shannon, Communication in the presence of noise. Proc. Inst. Radio Eng. 37, 10-21 (1949)

39. K Slattery, H Skinner, Platform Interference in Wireless Systems. (Elsevier, 2008)

\section{Submit your manuscript to a SpringerOpen ${ }^{\mathcal{O}}$ journal and benefit from:}

- Convenient online submission

- Rigorous peer review

- Immediate publication on acceptance

- Open access: articles freely available online

- High visibility within the field

- Retaining the copyright to your article

Submit your next manuscript at $\gg$ springeropen.com 